\title{
Overexpression of $\alpha$-Synuclein by Oligodendrocytes in Transgenic Mice Does Not Recapitulate the Fibrillar Aggregation Seen in Multiple System Atrophy
}

\author{
Florent Laferrière ${ }^{1,2,+}\left(\mathbb{D}, \mathrm{Xin}_{\mathrm{He}} \mathrm{1}^{1,2,3, \dagger}\right.$, Federica Zinghirino ${ }^{1,2,4} \mathbb{D}^{-}$, Evelyne Doudnikoff ${ }^{1,2}$, \\ Emilie Faggiani ${ }^{1,2}$, Wassilios G. Meissner ${ }^{1,2,5}$, Erwan Bezard ${ }^{1,2} \mathbb{D}$, Francesca De Giorgi ${ }^{1,2,6, *, \ddagger}$ \\ and François Ichas $1,2,6, *, \ddagger$ (D) \\ 1 CNRS, Institut des Maladies Neurodégénératives, UMR 5293, 33076 Bordeaux, France; \\ florent.laferriere@u-bordeaux.fr (F.L.); xin.he@u-bordeaux.fr (X.H.); federica.zinghirino@phd.unict.it (F.Z.); \\ evelyne.doudnikoff@u-bordeaux.fr (E.D.); emilie.faggiani@u-bordeaux.fr (E.F.); \\ wassilios.meissner@chu-bordeaux.fr (W.G.M.); erwan.bezard@u-bordeaux.fr (E.B.) \\ Institut des Maladies Neurodégénératives, UMR 5293, Université de Bordeaux, 33076 Bordeaux, France \\ Department of Neurology, Sheng Jing Hospital of China Medical University, Shenyang 110004, China \\ 4 Dipartimento di Scienze Biomediche e Biotecnologiche, BIOMETEC, Università degli Studi di Catania, \\ 95123 Catania, Italy \\ 5 Service de Neurologie, CRMR Atrophie Multisystématisée, CHU Bordeaux, 33000 Bordeaux, France \\ 6 INSERM, Laboratoire de Neurosciences Expérimentales et Cliniques, U-1084, Université de Poitiers, \\ 86000 Poitiers, France \\ * Correspondence: francesca.degiorgi-ichas@inserm.fr (F.D.G.); francois.ichas@inserm.fr (F.I.); \\ Tel.: +33-5-335-147-97 (F.D.G.); +33-6-627-527-19 (F.I.) \\ + These authors contributed equally to this work. \\ $\ddagger$ These authors contributed equally to this work as co-last authors.
}

Received: 30 June 2020; Accepted: 26 October 2020; Published: 29 October 2020

check for updates

\begin{abstract}
The synucleinopathy underlying multiple system atrophy (MSA) is characterized by the presence of abundant amyloid inclusions containing fibrillar $\alpha$-synuclein ( $\alpha$-syn) aggregates in the brains of the patients and is associated with an extensive neurodegeneration. In contrast to Parkinson's disease (PD) where the pathological $\alpha$-syn aggregates are almost exclusively neuronal, the $\alpha$-syn inclusions in MSA are principally observed in oligodendrocytes (OLs) where they form glial cytoplasmic inclusions (GCIs). This is intriguing because differentiated OLs express low levels of $\alpha$-syn, yet pathogenic amyloid $\alpha$-syn seeds require significant amounts of $\alpha$-syn monomers to feed their fibrillar growth and to eventually cause the buildup of cytopathological inclusions. One of the transgenic mouse models of this disease is based on the targeted overexpression of human $\alpha$-syn in OLs using the PLP promoter. In these mice, the histopathological images showing a rapid emergence of S129-phosphorylated $\alpha$-syn inside OLs are considered as equivalent to GCIs. Instead, we report here that they correspond to the accumulation of phosphorylated $\alpha$-syn monomers/oligomers and not to the appearance of the distinctive fibrillar $\alpha$-syn aggregates that are present in the brains of MSA or PD patients. In spite of a propensity to co-sediment with myelin sheath contaminants, the phosphorylated forms found in the brains of the transgenic animals are soluble $(>80 \%)$. In clear contrast, the phosphorylated species present in the brains of MSA and PD patients are insoluble fibrils ( $>95 \%)$. Using primary cultures of OLs from PLP- $\alpha$ Syn mice we observed a variable association of S129-phosphorylated $\alpha$-syn with the cytoplasmic compartment, the nucleus and with membrane domains suggesting that OLs functionally accommodate the phospho- $\alpha$-syn deriving from experimental overexpression. Yet and while not taking place spontaneously, fibrillization can be seeded in these primary cultures by challenging the OLs with $\alpha$-syn preformed fibrils (PFFs). This indicates that a targeted overexpression of $\alpha$-syn does not model GCIs in mice but that it can provide a basis for seeding aggregation using PFFs. This approach could help establishing a link between $\alpha$-syn aggregation and the development of a clinical phenotype in these transgenic animals.
\end{abstract}


Keywords: $\alpha$-synuclein; multiple system atrophy; GCIs

\section{Introduction}

Multiple system atrophy (MSA) is a fatal neurodegenerative disorder, characterized by a synucleinopathy that consists in the abundant presence of fibrillar $\alpha$-synuclein ( $\alpha$-syn) in oligodendrocytes (OLs) forming glial cytoplasmic inclusions (GCIs) and to a lesser extent in neurons [1-4]. Based on these observations and similar to other synucleinopathies like Parkinson's disease (PD), $\alpha$-syn self-assembly as amyloid fibrils is thought to represent a key pathologic event capable to account for the autocatalytic spread of the disease and thus represents a prominent candidate target for disease modification in MSA.

The origin of $\alpha$-syn in OLs remains the matter of an ongoing controversy [5]. During differentiation the OL progenitors progressively turn down $\alpha$-syn expression [6]. In addition and even if the presence of $\alpha$-syn mRNA has been reported in these cells, its levels are not statistically different in OLs from healthy vs. MSA brains [7]. Thus, the contribution of post-translational mechanisms has been proposed to account for the accumulation and the aggregation of $\alpha$-syn in OLs, among which: the relocation of the p25 $\alpha$ protein from the myelin sheath to the cytoplasm secondarily trapping $\alpha$-syn $[8,9]$ and alterations in the exosomal export of $\alpha$-syn causing its intracellular accumulation [10]. An alternative possibility is that in MSA $\alpha$-syn could be imported by OLs by virtue of a cell-to-cell transfer of $\alpha$-syn from neurons that strongly express $\alpha$-syn [11,12] and show abundant $\alpha$-syn oligomers in MSA patient brains [13].

Although it is unclear which is the origin of the pathological accumulation and aggregation of $\alpha$-syn in the OLs, most of the experimental animal models for MSA are based on a targeted overexpression of human $\alpha$-syn in these cells [14-16]. In these transgenic models the question of why and how $\alpha$-syn tends to accumulate in OLs is circumvented and only the events second to the experimental overexpression of $\alpha$-syn in OLs can be analyzed.

In spite of this shortcut, the attractiveness of these models lies in their capability to recapitulate certain key clinical and neurodegenerative features distinctive of MSA. They have thus been adopted to investigate the pathogenesis and pathophysiology of MSA, to identify new treatment targets and to validate the most promising compounds before clinical testing [5,17-21]. In addition, they also possibly offer an in vivo context to address the question of the causal link existing between the fibrillization of $\alpha$-syn, the development of neuropathological inclusions (GCIs) and the observed clinical phenotype. This causal relationship still remains unclear in MSA as well as in other synucleinopathies.

One of these transgenic mouse models uses the proteolipid protein promoter (PLP) to drive $\alpha$-syn expression in mice bred on a C57/BL6 background (PLP- $\alpha$ Syn) [14]. The brains of these mice exhibit a generalized OL $\alpha$-syn burden with extensive S129 phosphorylation [14]. Since this post-translational modification is associated with GCIs in MSA [22,23], it is thus widely accepted that this phosphorylated $\alpha$-syn burden indicates the emergence of "experimental GCIs" in this animal model [24]. This is seemingly in line with the observation that MSA-like clinical features (motor impairment, autonomic dysfunction) and neurodegeneration (i.e., DA neuron loss) develop in PLP- $\alpha$ Syn mice [25-29].

We thus put under closer scrutiny the $\alpha$-syn pools found in the OLs of these transgenic animals and compared these pools with the $\alpha$-syn extracted from the brains of control subjects, MSA and PD patients, as well as with synthetic preformed fibrils (PFFs) made of recombinant human $\alpha$-syn.

\section{Materials and Methods}

\subsection{Animals}

Mice expressing human $\alpha$-synuclein in oligodendrocytes under the control of the proteolipid promoter (PLP- $\alpha$ Syn) were previously generated on a C57BL/6J background [14] and kindly provided 
by Dr. P.O. Fernagut (University of Poitiers, Poitiers, France). Both PLP- $\alpha$ Syn and C57/BL6J mice were bred and maintained in the IMN animal facilities. All mice were housed in a temperature-controlled $\left(22^{\circ} \mathrm{C}\right)$ and light-controlled environment on a 12-h light/12-h dark cycle with access to food and water ad libitum. Animal sacrifice was conducted in accordance with the European Communities Council Directive (2010/63/EU) for care of laboratory animals

\subsection{Primary Culture of Cortical Neurons and High Content Analysis}

The list of chemicals appears in Supplementary Table 1. Timed pregnant mice were used. Brain cortices were harvested from E18 mouse embryos and dissociated enzymatically and mechanically (using the neuronal tissue dissociation kit, C-Tubes and an Octodissociator with heaters, Miltenyi Biotech, Bergisch Gladbach, Germany) to yield a homogenous cell suspension. The cells were then plated at 20,000 per well in 96-well plates (Corning, Biocoat Poly-D lysine Imaging plates, Corning, New York, NY, USA) in neuronal medium (Neuronal Macs medium, Miltenyi Biotech, Bergisch Gladbach, Germany) containing 0.5\% Penicillin/Streptomycin, $0.5 \mathrm{mM}$ alanyl-glutamine and 2\% Neurobrew supplement (Miltenyi Biotech, Bergisch Gladbach, Germany). The medium was changed by $1 / 3$ every 3 days, until a maximum of 30 days in vitro (DIV). When mentioned, isolated oligodendrocyte precursor cells (OPCs) (5000 per well) were added at DIV 2. For the induction of the experimental synucleinopathy, $10 \mathrm{nM}$ (equivalent monomeric concentration) of $\alpha$-syn PFFs were added at DIV 7 [30]. PFFs were templated on the 1B polymorph [30] (PFFs and PFFs\#1) or on the iso3 polymorph (PFF\#2) [30] by seeding the monomers with $1 \% w / w$ template prior to the fibrillization as described in Reference [30]. High Content Analysis was performed on multichannel fluorescence images acquired 20x using the generic analysis module of the Incucyte S3 and Top-Hat cellular segmentation was based on the fluorescence signal corresponding to the antibody of interest. For the quantification of the neuronal synucleinopathy we used the neurite segmentation module and based the recognition process on the fluorescence corresponding to the EP1536Y signal. This segmentation methodology is equivalent to the sequential application of two filters: one fluorescence-intensity-based that retains the EP1536Y-positive pixels above a certain threshold and one morphological that only retains the pixels groups forming linear objects (neurites). Settings were determined to allow the specific detection of the neuronal neuritic synucleinopathy and filter out the EP1536Y signal present elsewhere. For the sake of simplicity, we thus call it neuronal synucleinopathy in Figure 6. The parameters were as follows: Color Neurites panel (EP1536Y); cell-body cluster segmentation: Top Hat, Radius $20 \mu \mathrm{m}$, Threshold RCU 2; Cleanup: Min Cell Width 7 (other parameters 0); Cell-body cluster filter: none; Neurite Parameters: coarse sensitivity 10, fine sensitivity 0.5, Width $1 \mu \mathrm{m}$.

Table 1. List of antibodies used in the study.

\begin{tabular}{|c|c|c|c|c|c|}
\hline Antibody & Target & Company & Cat.No & Dilution IF & Dilution IB \\
\hline \multicolumn{6}{|c|}{ Primary antibodies } \\
\hline MJFR-1 & human alpha-synuclein & Abcam & ab138501 & 1: 1000 & 1: 10,000 \\
\hline EP1536Y & pS129 phospho-synuclein & Abcam & ab51253 & 1: 500 & 1: 5000 \\
\hline Syn 1 clone 42 & $\begin{array}{l}\text { human and murine } \\
\text { alpha-synuclein }\end{array}$ & BD Biosciences & 610787 & 1: 500 & 1: 2000 \\
\hline D37A6 & murine alpha-synuclein & Cell Signaling & \#4179 & 1: 200 & 1: 2000 \\
\hline SynF1 & $\begin{array}{c}\text { aggregated } \\
\text { alpha-synuclein }\end{array}$ & BioLegend & 847802 & 1: 500 & 1: 10,000 \\
\hline syn211 & human alpha-synuclein & Abcam & ab80627 & 1: 500 & $\mathrm{n} / \mathrm{a}$ \\
\hline MBP & myelin basic protein & Abcam & ab218011 & 1: 200 & 1: 2000 \\
\hline CNPase & cyclic nucleotide phospho. & Abcam & ab6319 & 1: 500 & $\mathrm{n} / \mathrm{a}$ \\
\hline Sox10 & sox 10 protein & Abcam & ab155279 & 1: 200 & $\mathrm{n} / \mathrm{a}$ \\
\hline NeuN & neuronal nuclei protein & $\begin{array}{c}\text { Merck } \\
\text { Millipore }\end{array}$ & MAB377 & 1: 500 & $\mathrm{n} / \mathrm{a}$ \\
\hline Actin & beta-actin & Sigma & A5316 & $\mathrm{n} / \mathrm{a}$ & 1: 10,000 \\
\hline
\end{tabular}


Table 1. Cont.

\begin{tabular}{|c|c|c|c|c|c|}
\hline Antibody & Target & Company & Cat.No & Dilution IF & Dilution IB \\
\hline \multicolumn{6}{|c|}{ Secondary antibodies } \\
\hline Goat anti-mouse HRP & mouse $\operatorname{IgG}(\mathrm{H}+\mathrm{L})$ & $\begin{array}{l}\text { Jackson } \\
\text { Immuno }\end{array}$ & $115-035-146$ & $\mathrm{n} / \mathrm{a}$ & 1: 10,000 \\
\hline Goat anti-rabbit HRP & rabbit $\operatorname{IgG}(\mathrm{H}+\mathrm{L})$ & $\begin{array}{l}\text { Jackson } \\
\text { Immuno }\end{array}$ & $111-035-144$ & $\mathrm{n} / \mathrm{a}$ & 1: 10,000 \\
\hline $\begin{array}{l}\text { Goat anti-mouse } \\
\text { IRDye 680RD }\end{array}$ & mouse $\operatorname{IgG}(\mathrm{H}+\mathrm{L})$ & LI-COR & $926-68070$ & $\mathrm{n} / \mathrm{a}$ & 1: 5000 \\
\hline $\begin{array}{c}\text { Goat anti-rabbit IRDye } \\
800 \mathrm{CW}\end{array}$ & rabbit $\operatorname{IgG}(\mathrm{H}+\mathrm{L})$ & LI-COR & $926-32211$ & $\mathrm{n} / \mathrm{a}$ & 1: 5000 \\
\hline $\begin{array}{c}\text { Donkey anti-mouse } \\
\text { Alexa } 488\end{array}$ & mouse IgG $(\mathrm{H}+\mathrm{L})$ & $\begin{array}{l}\text { Thermo Fisher } \\
\text { Sci. }\end{array}$ & A-21202 & 1: 500 & $\mathrm{n} / \mathrm{a}$ \\
\hline $\begin{array}{c}\text { Goat anti-rabbit Alexa } \\
488\end{array}$ & rabbit $\operatorname{IgG}(\mathrm{H}+\mathrm{L})$ & $\begin{array}{l}\text { Thermo Fisher } \\
\text { Sci. }\end{array}$ & A-11008 & 1: 500 & $\mathrm{n} / \mathrm{a}$ \\
\hline $\begin{array}{c}\text { Donkey anti-mouse } \\
\text { Alexa } 594\end{array}$ & mouse $\operatorname{IgG}(\mathrm{H}+\mathrm{L})$ & $\begin{array}{l}\text { Thermo Fisher } \\
\text { Sci. }\end{array}$ & A-21203 & 1: 500 & $\mathrm{n} / \mathrm{a}$ \\
\hline $\begin{array}{c}\text { Donkey anti-rabbit } \\
\text { Alexa } 594\end{array}$ & rabbit $\operatorname{IgG}(\mathrm{H}+\mathrm{L})$ & $\begin{array}{l}\text { Thermo Fisher } \\
\text { Sci. }\end{array}$ & A-21207 & 1: 500 & $\mathrm{n} / \mathrm{a}$ \\
\hline
\end{tabular}

IF: Immunofluorescence; IB: Immunoblotting.

\subsection{Primary Culture of Mouse Oligodendrocytes}

Timed pregnant mice were used. Brain cortices were harvested from E18 mouse embryos and dissociated enzymatically and mechanically (using Neuronal tissue dissociation kit, C-Tubes and Octodissociator with heaters, all from Miltenyi Biotech, Bergisch Gladbach, Germany) to yield a homogenous cell suspension.

Oligodendrocyte precursor cells were then isolated from this suspension based on the expression

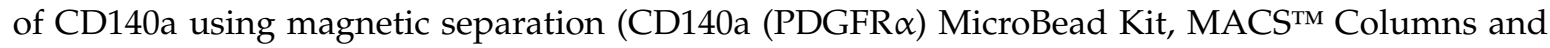
MACS $^{\mathrm{TM}}$ Separators, all from Miltenyi Biotech, Bergisch Gladbach, Germany) using the protocol made available by Miltenyi Biotech.

The cells were then plated at 5000 per well in 96-well Biocoat plates (poly L-lysine, Corning, New York, NY, USA) in neuronal medium (Neuronal Macs medium, Miltenyi Biotech, Bergisch Gladbach, Germany) containing $0.5 \%$ penicillin-streptomycin, $0.5 \mathrm{mM}$ alanyl-glutamine and $2 \%$ Neurobrew supplement (Miltenyi Biotech, Bergisch Gladbach, Germany), FGF 20 ng/mL, PDGF-A $20 \mathrm{ng} / \mathrm{mL}$ (Miltenyi Biotech, Bergisch Gladbach, Germany). Medium was changed every 3 days by one third. From DIV 7 on, medium was progressively replaced by Brainphys (Stemcell Technologies, Vancouver, BC, Canada) supplemented with Neurobrew 2\%, FGF 20 ng/mL, PDGF-A 20 ng/mL. At DIV 26 PDGF-A was omitted and replaced with CTNF $10 \mathrm{ng} / \mathrm{mL}$ (Miltenyi Biotech, Bergisch Gladbach, Germany) for differentiation.

\subsection{Recombinant $\alpha$-Syn Expression and Purification}

E. coli strain BL21 (DE3) plysS was transformed with pET24- $\alpha$-Syn vector by electroporation and plated onto LB agar plate containing $30 \mu \mathrm{g} / \mathrm{mL}$ Kanamycin. A pre-culture in $5 \mathrm{~mL}$ LB medium was inoculated with one clone and incubated at $37^{\circ} \mathrm{C}$ under $200 \mathrm{rpm}$ shaking for $4 \mathrm{~h}$. The expression on $\alpha$-syn was carried out in M9 minimal medium containing $2 \mathrm{~g} / \mathrm{L}$ of $13 \mathrm{C}$ Glucose and $1 \mathrm{~g} / \mathrm{L}$ of $\mathrm{NH}_{4} \mathrm{Cl}$ as carbon and nitrogen sources. Cells from LB pre-culture were recovered by centrifugation $(1000 \times g-10 \mathrm{~min})$ and used for inoculating $200 \mathrm{~mL}$ of M9 medium. Cells were grown overnight at $37^{\circ} \mathrm{C}$ under $200 \mathrm{rpm}$ shaking and then diluted in $2 \mathrm{~L}$ of culture. Protein expression was induced by adding $1 \mathrm{mM}$ IPTG during exponential phase, evaluated by Optical Density at $600 \mathrm{~nm}$ reaching 0.8 . Cells were harvested after $4-5 \mathrm{~h}$ of culture at $37^{\circ} \mathrm{C}$ by $6000 \times g$ centrifugation (JLA 8.1 Beckman Coulter) and pellet was kept at $-20^{\circ} \mathrm{C}$ before purification.

Pellet was thawed in $10 \mathrm{mM}$ Tris- $\mathrm{HCl}$ (pH 8.0), $1 \mathrm{mM}$ EDTA and $1 \mathrm{mM}$ PMSF, Pierce Complete EDTA-free protease inhibitors tablet (Thermofisher) buffer and sonicated 3 times for $45 \mathrm{sec}$ (Bandelin 
Sonoplus-VS70T probe) prior to be centrifuging. The supernatant was boiled for $20 \mathrm{~min}$ and centrifuged. Streptomycin sulphate was added to supernatant to a final concentration of $10 \mathrm{mg} / \mathrm{mL}$ and the solution was stirred for $15 \mathrm{~min}$ at $4{ }^{\circ} \mathrm{C}$ and then centrifuged. Ammonium sulphate was added to supernatant to a final concentration of $360 \mathrm{mg} / \mathrm{mL}$ and the mixture was stirred for $15 \mathrm{~min}$ at $4{ }^{\circ} \mathrm{C}$ before being centrifuged. These four centrifugations were performed at 20,000 rpm for $30 \mathrm{~min}$ and at $4{ }^{\circ} \mathrm{C}$ with Beckman Coulter JA-25.5 rotor. The pellet was resuspended in $25 \mathrm{mM}$ Tris- $\mathrm{HCl}(\mathrm{pH} \mathrm{7.70)}$ and dialyzed against the same buffer to eliminate salts. The dialyzed sample was injected onto HiTrap Q HP column previously equilibrated with $25 \mathrm{mM}$ Tris- $\mathrm{HCl}$ (pH 7.70) and $\alpha$-synuclein was eluted around $250 \mu \mathrm{M}$ of $\mathrm{NaCl}$ by steps from $0 \mathrm{mM}$ to $500 \mathrm{mM} \mathrm{NaCl}$ with AKTA pure system. Fractions containing the protein were dialyzed against $20 \mathrm{mM}$ Tris- $\mathrm{HCl}$ (pH 7.40) and $100 \mathrm{mM} \mathrm{NaCl}$ buffer before to be loading onto HiLoad 26/600 Superdex $75 \mathrm{pg}$ column equilibrated with the same buffer with AKTApur system. Monomeric fractions were collected and concentrated if needed by using Vivaspin 15R $2 \mathrm{kDa}$ cut off concentrator (Sartorius Stedim, Gottingen, Germany). Purification fractions were checked by using Poly Acrylamide Gel Electrophoresis Tris-tricine 13\% dying with ProBlueSafe Strain. Protein concentration was evaluated spectrophotometrically by using absorbance at $280 \mathrm{~nm}$ and extinction coefficient of $5960 \mathrm{M}^{-1} . \mathrm{cm}^{-1}$.

\section{5. $\alpha$-syn Fibrillization}

Solutions of monomeric $\alpha$-syn at $4-5 \mathrm{mg} / \mathrm{mL}$ were sterilized by filtration with $0.22 \mu \mathrm{m}$ Millipore single use filters and stored in sterile $15 \mathrm{~mL}$ conical falcon tubes at $4{ }^{\circ} \mathrm{C}$. Sterilized stock was then distributed into safe-lock "Biopur" individually sterile-packaged $1.5 \mathrm{~mL}$ Eppendorf tubes as $500 \mu \mathrm{L}$ aliquots. The tubes were cap-locked and additionally sealed with parafilm. All the previous steps were performed aseptically in a particle-free environment, under a microbiological safety laminar flow hood. For comparative fibrillizations, all the samples were loaded simultaneously in a Thermomixer (Eppendorf, Hamburg, Germany) in a 24 position $1.5 \mathrm{~mL}$ Eppendorf tube holder equipped with a heating lid. Temperature was set to $37^{\circ} \mathrm{C}$ and shaking to $2000 \mathrm{rpm}$. Sampling for measurements during the fibrillization process was done by temporarily returning the samples under the microbiological safety laminar flow hood.

Samples from the fibrillized $\alpha$-syn aliquots were diluted to $0.1 \mathrm{mg} / \mathrm{mL}$ in $100 \mu \mathrm{L}$ in PBS and distributed in cap-locked sterile $0.5 \mathrm{~mL}$ PCR tubes (Thermo Fisher Scientific, Waltham, MA, USA). Sonication was performed at $25^{\circ} \mathrm{C}$ in a BioruptorPlus water bath sonicator (Diagenode, Seraing, Belgium) equipped with thermostatic control and automated tube carousel rotator. The sonication power was set to "high" and 10 cycles of $30 \mathrm{~s}$ "on" followed by $10 \mathrm{~s}$ "off" were applied.

\subsection{Immunofluorescence}

Cells were fixed with $4 \%(w / v)$ paraformaldehyde/ $4 \%(w / v)$ sucrose for $15 \mathrm{~min}$, permeabilized and blocked with $3 \%(w / v)$ BSA/0.1\% (vol/vol) TX-100 for $15 \mathrm{~min}$ and incubated at $4{ }^{\circ} \mathrm{C}$ overnight with primary antibody diluted in the blocking buffer (Table 1 ). Secondary antibody was incubated for $1 \mathrm{~h}$ at $37^{\circ} \mathrm{C}$ (Table 1).

\subsection{Brain Sections Staining}

The brains were perfused with saline, post-fixed for 3 days in $10 \mathrm{~mL}$ of $4 \%$ paraformaldehyde at $4{ }^{\circ} \mathrm{C}$, cryoprotected in gradient $20 \%$ sucrose in PBS before being frozen by immersion in a cold isopentane bath $\left(-60^{\circ} \mathrm{C}\right)$ for at least $5 \mathrm{~min}$ and stored immediately at $-80^{\circ} \mathrm{C}$ until sectioning for immunofluorescence analysis. After serial sectioning, the sections were stained using the primary antibodies EP1536Y (Abcam) for detecting phospho-S129 positive $\alpha$-syn and MJFR1 for human $\alpha$-syn. The slides were acquired using Incucyte S3 High Content Imager (Sartorius, Göttingen, Germany) with a home-made 3D-printed slide holder for IF. 


\subsection{SarkoSpin Fractionation}

SarkoSpin procedure was slightly adapted from previously published protocols [31,32]. The samples were obtained from brains collected in a Brain Donation Program of the Brain Bank "GIE NeuroCEB" (Neuro-CEB BB-0033-00011). The consents were signed by the patients themselves or their next of kin in their name, in accordance with the French Bioethical Laws. The Brain Bank GIE NeuroCEB has been declared at the Ministry of Higher Education and Research and has received approval to distribute samples (agreement AC-2013-1887). Human cortices (cingulate gyrus) were dissected from freshly frozen post-mortem brain samples from $n=3$ control, sporadic PD or MSA subjects respectively. For mouse brain samples, at the age indicated, mice were anesthetized and intracardially perfused with $0.9 \%$ saline. Brains were quickly removed and homogenized. Mouse and human brain tissues were homogenized at $10 \%(w / v)$ in solubilization buffer (SB): $10 \mathrm{mM}$ Tris pH 7.5, $150 \mathrm{mM} \mathrm{NaCl}, 0.1 \mathrm{mM}$ EDTA, $1 \mathrm{mM}$ DTT, Complete EDTA-free protease inhibitors (Roche, Basel, Switzerland) and PhosSTOP phosphatase inhibitors (Roche) using a gentleMACS Octo Dissociator (Miltenyi Biotec, Bergisch Gladbach, Germany) with M Tubes and the Protein extraction program. Stock preparations of $\alpha$-syn amyloid fibrils ( $5 \mathrm{mg} / \mathrm{mL}$ in TBS) were diluted with SB buffer to obtain $1 \mu \mathrm{g}$ in each centrifugation sample in an equivalent volume to brain homogenates. Samples were mixed 1:1 with SB $4 \%$ or $2 \%(w / v)$ N-lauroyl-sarcosine (sarkosyl, Sigma-Aldrich, Saint-Louis, MO, USA) for human brain or mouse brain and PFF respectively, 2 U. $\mu \mathrm{L}^{-1}$ Benzonase (Novagen, Burlington, $\mathrm{MA}, \mathrm{USA}$ ) and $4 \mathrm{mM} \mathrm{MgCl}_{2}$, reaching a final volume of $500 \mu \mathrm{L}$. SarkoSpin solubilization was then performed by incubating the samples at $37^{\circ} \mathrm{C}$ under constant shaking at $600 \mathrm{rpm}$ (Thermomixer, Eppendorf, Hamburg, Germany) for $45 \mathrm{~min}$. Solubilized samples were then mixed 1:1 with SB 40\% $(w / v)$ sucrose, without sarkosyl $\mathrm{MgCl}_{2}$ and Benzonase, in $1 \mathrm{~mL}$ polycarbonate ultracentrifuge tubes (Beckman, Brea, CA, USA) and centrifuged at 250,000 $\mathrm{g}$ for $1 \mathrm{~h}$ at room temperature with a TLA 120.2 rotor using an Optima XP benchtop ultracentrifuge (Beckman, Brea, CA, USA). Supernatant were collected by pipetting. Pellets were resuspended directly in the tube with $100 \mu \mathrm{L}$ of the buffer corresponding to the supernatant (SB 0.5 or $1 \%$ sarkosyl and 0 or $20 \%$ sucrose) and mixed with the same buffer in a fresh tube for reaching $1 \mathrm{ml}$ (equal volumes to supernatant).

\subsection{Sedimentation Velocity Gradient Fractionation}

Sedimentation velocity gradient fractionations were performed as published previously [31-33]. Briefly, a volume of $400 \mu \mathrm{L}$ of SarkoSpin solubilized samples was loaded on top of a $11 \mathrm{~mL}$ continuous $5-20 \%$ iodixanol gradient (Optiprep, 60\% (w/v) iodixanol, Sigma-Aldrich, Saint-Louis, MO, USA) in SB buffer with $0.5 \%(w / v)$ sarkosyl linearized directly in ultracentrifuge $12 \mathrm{~mL}$ tubes (Seton scientific, Petaluma, CA, USA) with a Gradient Master (Biocomp instruments, Fredericton, NB, Canada). The gradients were centrifuged at $200,000 \times g$ for $2.5 \mathrm{~h}$ at room temperature in a swinging-bucket SW-41 Ti rotor using an Optima LE-80K ultracentrifuge (Beckman, Brea, CA, USA). Gradients were then segregated into 16 equal fractions from the top using a piston fractionator (Biocomp instruments, Fredericton, NB, Canada) and a fraction collector (Gilson, Middleton, WI, USA). Fractions were aliquoted for further analysis of their content by immunoblot. Gradient linearity was verified by refractometry.

\subsection{Analysis of the Protein Contents of Sarkospin and Velocity Fractions by Filter Trap}

For filter trap assays, native fractions were spotted onto nitrocellulose $0.2 \mu \mathrm{m}$ membranes (Protran, GE) using a dot blot vacuum device (Whatman, Maidstone, UK). Nitrocellulose membranes were fixed for $30 \mathrm{~min}$ in PBS with PFA 0.4\% (v/v) (Sigma-Aldrich, Saint-Louis, MO, USA) final concentration. After three washes with PBS, membranes were blocked with $5 \%(w / v)$ skimmed powder milk in PBS-Tween $0.5 \%(v / v)$ and probed with primary and secondary antibodies in PBS-Tween with $4 \%(w / v)$ BSA (Table 1). Immunoreactivity was whether visualized by chemiluminescence or infrared using 
Clarity ECL and Chemidoc (Biorad, Hercules, CA, USA) or Odissey systems (Li-Cor, Lincoln, NE, USA)) respectively.

\subsection{Crosslinking and Western Blot}

Pooled ( $n=3$ ) WT or PLP- $\alpha$ Syn mouse brain homogenates were prepared as described above. Samples of equal protein concentration (determined with BCA) were denucleated by mild centrifugation at $800 \times g$ for $5 \mathrm{~min}$ at $4{ }^{\circ} \mathrm{C}$. Supernatants were treated $15 \mathrm{~min}$ at room temperature $1 \mathrm{U}_{\mathrm{H}} \mu \mathrm{L}^{-1}$ Benzonase (Novagen, Burlington, MA, USA) with $2 \mathrm{mM} \mathrm{MgCl}_{2}$ final concentrations. When specified, samples were solubilized $30 \mathrm{~min}$ on ice with SB $0.25 \%(v / v)$ Triton-X final concentration, equal volumes of SB without detergent was added to non-solubilized samples incubated under the same conditions. When specified, samples were crosslinked with $2 \mathrm{mM}$ final concentration disuccinimidyl glutarate (DSG, Sigma) at room temperature for $15 \mathrm{~min}$ before stopping the reaction with $100 \mathrm{mM}$ final concentration of Tris. Non-crosslinked samples were treated in the same conditions without DSG. Aliquots of the resulting samples were added Laemmli $1 \mathrm{X}$ prior to denaturation at $95^{\circ} \mathrm{C}$ for $5 \mathrm{~min}$ and loaded on Mini-Protean TGX $12 \%$ gels (Biorad) followed by SDS-PAGE electrophoresis. Gels were transferred on nitrocellulose $0.2 \mu \mathrm{m}$ membranes with Trans-Blot Turbo transfer system (Biorad) using the Mixed molecular weight program. Membranes were fixed with PFA and proteins were immunolabelled with infrared secondary antibodies as described for filter trap. After detection with Odyssey system (Li-Cor), whole lane signal intensity profiles were quantified and plotted on graphs with MetaMorph software (Molecular Devices, San José, CA, USA).

\subsection{Antibodies}

All the antibodies used and shown in the study are summarized in Table 1. All the plates were acquired and analyzed using an Incucyte S3 High Content Imager (Sartorius, Göttingen, Germany).

\section{Results and Discussion}

3.1. S129-phosphorylated $\alpha$-syn Species Found in the Brain of PLP- $\alpha$ Syn Mice Are Distinct from the Amyloid forms Extracted from PD and MSA Brains and from Recombinant PFFs

As previously reported using immunohistochemistry [14], double immunofluorescence (Figure 1A) revealed that the OLs populating the brains of PLP- $\alpha$ Syn mice express high levels of human $\alpha$-syn (Figure 1A, MJFR1 panel). Indicative of a massive overexpression, human $\alpha$-syn levels in OLs are so high that they account on their own for total brain $\alpha$-syn levels $500 \%$ to $800 \%$ higher than in wild-type animals [34]. Thus, image acquisition adapted to detect $\alpha$-syn in the OLs of PLP- $\alpha$ Syn mice with the species-independent antibody syn1 (Figure 1A, syn1 panels) leaves the endogenous physiological $\alpha$-syn neuronal signal in the background. Similarly, these acquisition conditions for syn 1 also yield a completely dark image for control animals (Figure 1A). In PLP- $\alpha$ Syn mice most of the OLs are also positive for EP1536Y that detects S129-phosphorylated $\alpha$-syn (Figure 1A, EP1536Y panel). In these conditions brain sections from WT animals are totally negative for EP1536Y (Figure 1A). 
A
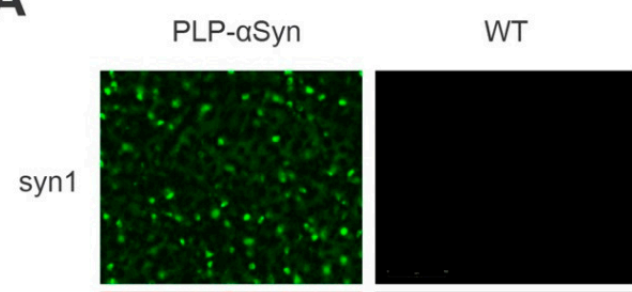

(6 months)

PLP-aSyn

WT
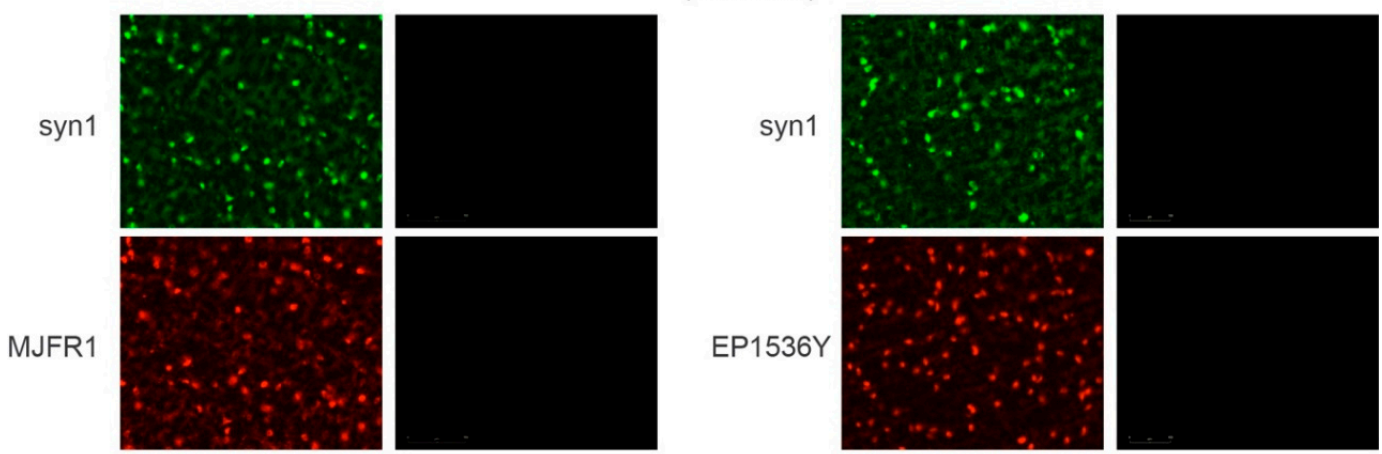

merge
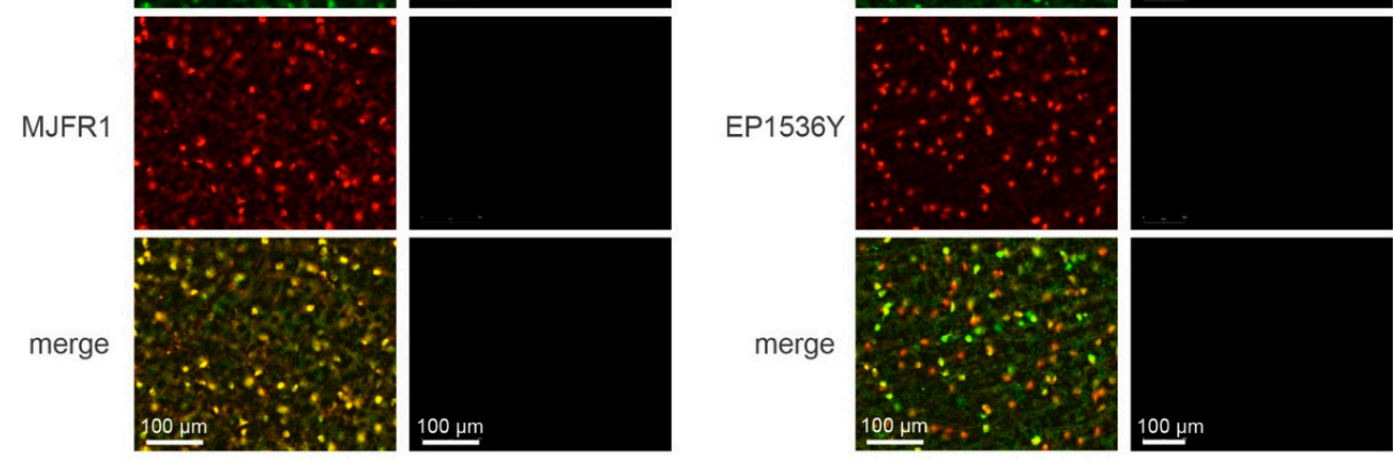

MJFR1
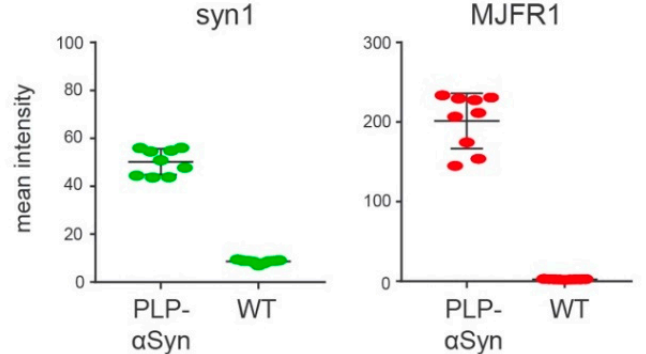

B
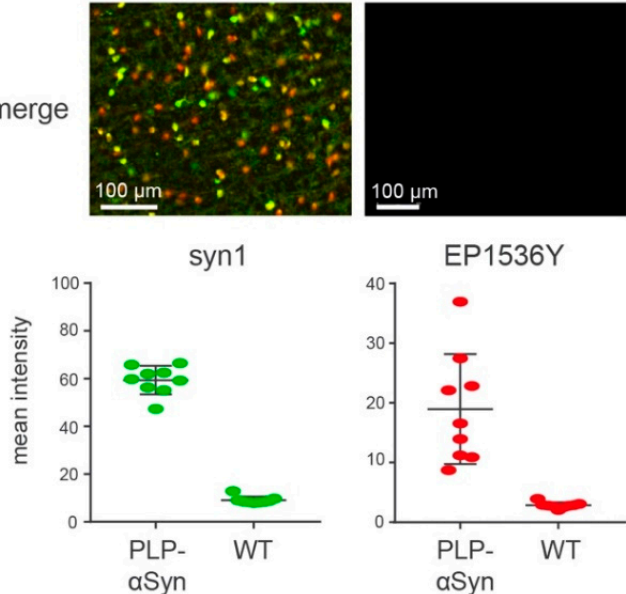

EP1536Y

$B$

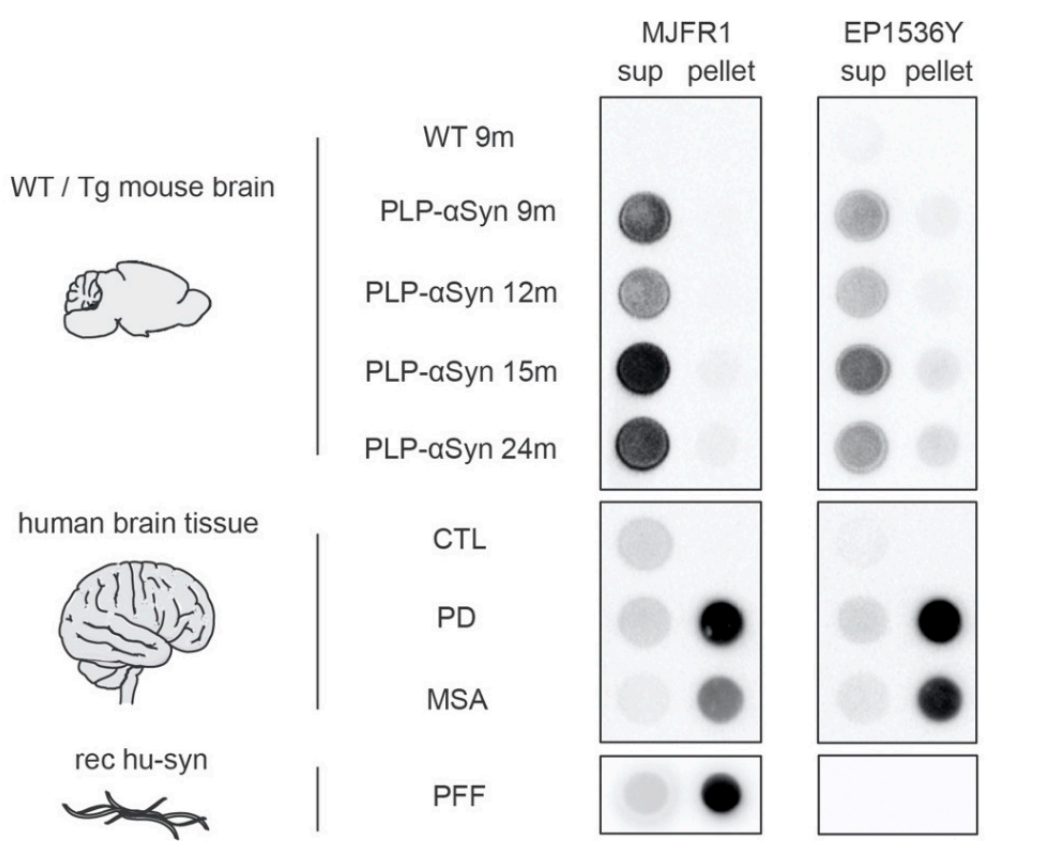

Figure 1. The S129-phosphorylated $\alpha$-syn species found in the brain of PLP- $\alpha$ Syn mice are distinct from the amyloid forms extracted from Parkinson's disease (PD) and multiple system atrophy (MSA) brains and from recombinant preformed fibrils (PFFs). (A) Immunofluorescence staining of 6 months-old PLP- $\alpha$ Syn mouse cortical sections, representative of 3 independent experiments. Total $\alpha$-syn (syn1, green, top panels) shows a complete colocalization with human $\alpha$-syn (MJFR1, red, mid-left panels) and partial colocalization with pS129 phosphorylated $\alpha$-syn (EP1536Y, red, mid-right panels). The 4 lower panel quantifications depict the mean field intensity values of 9 cortical/striatal fields of views at $20 \times$ 
from the sections shown above and confirm the massive overexpression of $\alpha$-syn in PLP- $\alpha$ Syn mouse brains compared to wild-type (WT). (B) Biochemical aggregation analysis of the different species of $\alpha$-syn found in WT or PLP- $\alpha$ Syn mouse brains (top), control, PD and MSA human subject brains (middle) or a preparation of recombinant human $\alpha$-syn PFF (bottom). Pooled 9 to 24 months-old mice $(n=3)$ or human $(n=3)$ brain homogenates and PFF samples were subjected to SarkoSpin procedure consisting of a sarkosyl solubilization at $37^{\circ} \mathrm{C}$ with nuclease under shaking followed by an ultracentrifugation on sucrose cushion. The contents in human $\alpha$-syn (MJFR1, left panel) and pS129- $\alpha$-syn (EP1536Y, right panel) of SarkoSpin supernatant and pellet fractions were assessed by filter trap followed by immunolabelling with the respective antibodies. Pictures are representative of $n=3$ independent Sarkospin procedures quantified in Supplementary Figure S1.

The emergence of hyperphosphorylated $\alpha$-syn in the OLs of PLP- $\alpha$ Syn mice was previously interpreted as the formation of "GCIs" [14,34]. GCIs are cytoplasmic inclusions found in the OLs in the brains of MSA patients that contain fibrillar $\alpha$-syn [35,36]. We thus proceeded with parallel extractions, sedimentations and filter-trapping of sarkosyl-insoluble amyloid aggregates (see methods) (Figure 1B) from the brains (i) of transgenic animals at different ages (Figure 1B, PLP- $\alpha$ Syn), (ii) of control animals (Figure 1B, WT), (iii) of human control, PD and MSA subjects (Figure 1B, CTL, PD, MSA), as well as from pure recombinant human $\alpha$-syn preformed fibrils (Figure 1B, PFF).

The results indicate that the method efficiently separated $\alpha$-syn amyloid fibrils from soluble $\alpha$-syn forms which coincided with the S129-phosphorylated $\alpha$-syn pool for PD and MSA brain extracts (see methods) (Figure 1B, PFF, PD, MSA). Indeed, by revealing human $\alpha$-syn with the MJFR1 antibody, it appeared that over $70 \%$ of the total $\alpha$-syn present in brain extracts from PD and MSA patients was pelleted and trapped, over $90 \%$ when synthetic PFFs were used and less than $3 \%$ when dealing with brain extracts from control subjects. In PD and MSA patients, S129-phosphorylated $\alpha$-syn coincided with the insoluble $\alpha$-syn pool since more than $85 \%$ of the phospho-S129 signal (EP1536Y-positive) was found in the pellets. In clear contrast, this figure was less than $3 \%$ for the control subjects (Supplementary Figure S1)

In strong contrast to MSA or PD brain extracts however, the same procedure applied to samples from PLP- $\alpha$ Syn mice showed that the fraction of $\alpha$-syn that could be pelleted and trapped was unexpectedly low, remaining below 7\% (Supplementary Figure S1). This indicated that the human $\alpha$-syn burden that builds up in these animals is not fibrillar like in PD or MSA. Strikingly enough and again in contrast with PD and MSA, the high levels of S129-phosphorylated $\alpha$-syn present in the brains of the PLP- $\alpha$ Syn mice appeared to correspond to soluble forms as over $80 \%$ of the latter were retained in the supernatants while only $3 \%$ to $5 \%$ was retained in the supernatants for PD and MSA samples (Supplementary Figure S1).

\subsection{S129-phosphorylated Human $\alpha$-syn Found in PLP- $\alpha$ Syn Mouse Brains Corresponds to Soluble Monomeric and Oligomeric Forms}

In order to better characterize the nature of the latter $\alpha$-syn pool, we proceeded with velocity sedimentations on linear iodixanol gradients that finely separate the $\alpha$-syn assemblies present in the samples according to size and density (Figure 2A and Supplementary Figure S2). The results confirmed that the phospho S129-positive OL $\alpha$-syn pool present in PLP- $\alpha$ Syn brains is not fibrillar like in PD or MSA patients. Instead, it sediments at the same levels as the unphosphorylated physiological forms of $\alpha$-syn found in control human brains (Figure 2A and Supplementary Figure S2), that is, soluble monomers and oligomers. Regarding the pS129-positive $\alpha$-syn pool present in OLs, SDS-PAGE performed on PLP- $\alpha$ Syn mouse brain extracts only evidenced pS129-positive monomers (Figure 2B). However, prior crosslinking of the extracts with DSG to prevent oligomer disassembly by SDS, allowed the identification of an oligomeric population (Figure 2B), confirming the results obtained in velocity sedimentation experiments (Figure 2A). Interestingly, these oligomers were no longer detected when Triton-X was applied before crosslinking (Figure 2B). With regards to this result, structuration of 
the oligomers around lipids and/or presence of weak hydrophobic interactions facilitating oligomer assembly can be hypothesized.

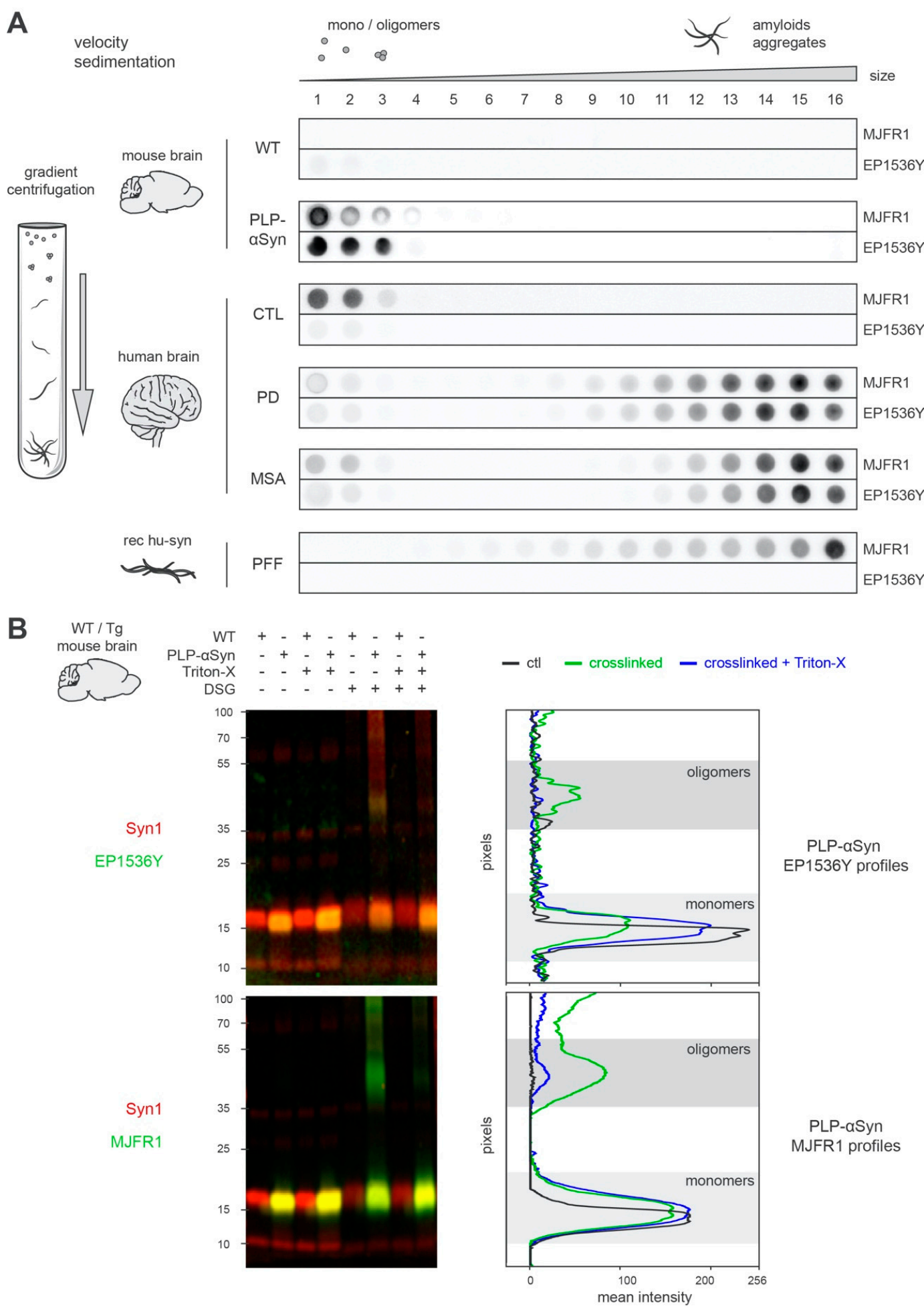

Figure 2. Unlike in MSA and PD, S129-phosphorylated human $\alpha$-syn is oligomeric and monomeric in PLP- $\alpha$ Syn mouse brains. (A) Aggregation profiles obtained by sedimentation of the different species of $\alpha$-syn found in WT or PLP- $\alpha$ Syn mouse brains (top), control, PD and MSA human subject brains (middle) or a preparation of recombinant human $\alpha$-syn PFF (bottom). Pooled mouse $(n=3)$ or human $(n=3)$ brain homogenates and PFF samples were subjected to SarkoSpin solubilization followed with 
fractionation by sedimentation velocity upon ultracentrifugation on iodixanol gradient. The distribution of human $\alpha$-syn (MJFR1) and pS129- $\alpha$-syn (EP1536Y) was analyzed by filter trap on the collected fractions (numbered from top to bottom of gradient) followed by immunostaining with the respective antibodies. Pictures are representative of $n=3$ independent sedimentations quantified in Supplementary Figure S2. (B) Representative western blot pictures (left) and their intensity quantification (right) of WT/PLP- $\alpha$ Syn mouse brains samples. Cytosolic fractions from pooled 9 months-old WT $(n=3)$ or age-matched PLP- $\alpha$ Syn $(n=3)$ brain homogenates were treated or not with Triton-X $(0.25 \%, 30 \mathrm{~min}$ on ice) followed by crosslinking or not with DSG ( $2 \mathrm{mM}$, room temperature). These samples were subjected to SDS-PAGE electrophoresis and immunoblotted with couples of antibodies directed against total $\alpha$-syn (syn1, red) and pS129- $\alpha$-syn (EP1536Y, green, top) or human $\alpha$-syn (MJFR1, green, bottom) using infrared dyes labelled secondary antibodies. Signal intensity of pS129- $\alpha$-syn (top) and human $\alpha$-syn (bottom) were quantified vertically by line scanning for PLP- $\alpha$ Syn samples untreated (black), DSG-crosslinked (green) or with prior Triton-X treatment (blue). Monomeric (light) and oligomeric (dark) species of the two forms of the proteins are depicted with the grey zones.

Collectively these results indicate that in PLP- $\alpha$ Syn mice (i) fibrillar forms of $\alpha$-syn are not detectable and (ii) that the pS129-positive OL $\alpha$-syn pool corresponds to soluble monomers and oligomers and cannot thus be considered as GCIs.

With regard to their localization in OLs and to the impact of Triton-X, we reasoned that a possibility could be that the pS129-positive monomers and oligomers seen in the transgenic animals could be associated with myelin. To address this hypothesis, we simply ran again the ultracentrifugation separation experiments shown in Figure 1B but removing this time the sucrose cushion meant to float the myelin sheath contaminants present in the brain extracts (Figure 3A). As expected, Figure 3A shows that omission of the sucrose cushion in the separation procedures resulted in a drastic enrichment of the pellet fraction with myelin basic protein (MBP) indicative of myelin sheath sedimentation. The presence of the latter myelin contaminants coincided with the appearance and the reinforcement of a pellet signal (i) for EP1536Y that detects S129-phosphorylated $\alpha$-syn, (ii) for the anti-synuclein antibodies syn1 and MJFR1 both capable of detecting the human $\alpha$-syn overexpressed by the OLs, (iii) but not for D37A6 that detects only the endogenous mouse $\alpha$-syn expressed in neurons. Quantifications of the dot blots shown in Figure 3A are shown in Supplementary Figure 3. These results are compatible with the idea that the pS129-positive monomers and oligomers seen in the transgenic animals can get associated with myelin. They also suggest that previous studies in which no specific care was taken to manage myelin contaminants could have been confounded, the latter "dragging along" soluble monomer and oligomers of $\alpha$-syn into the pellet.

Interestingly, the notion of association with myelin is also supported by the immunofluorescence mapping of the pS129-positive $\alpha$-syn pool in brain sections of the transgenic animals (Figure 3B). Indeed, a very strong and diffuse increase of EP1536Y signal is observable in regions rich in myelinated axons like the corpus callosum and the anterior commissure (Figure 3B). 
A

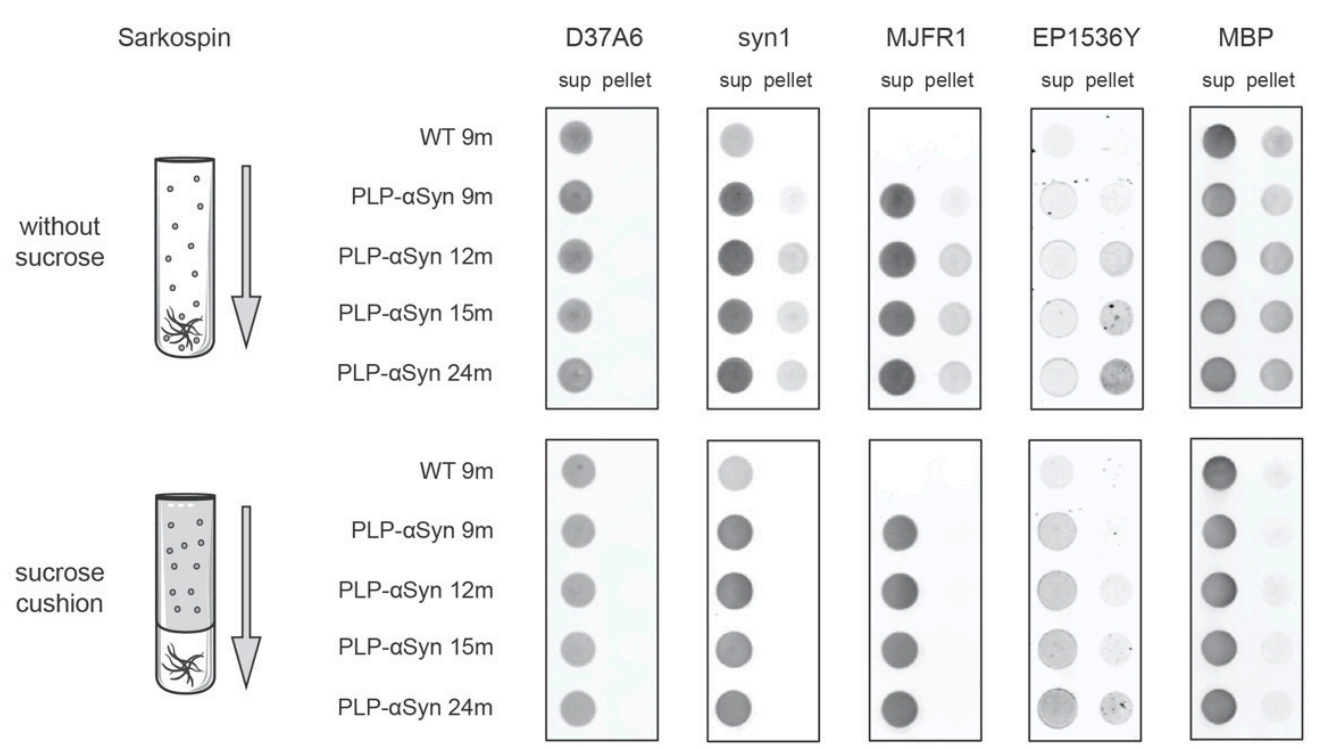

B

EP1536Y
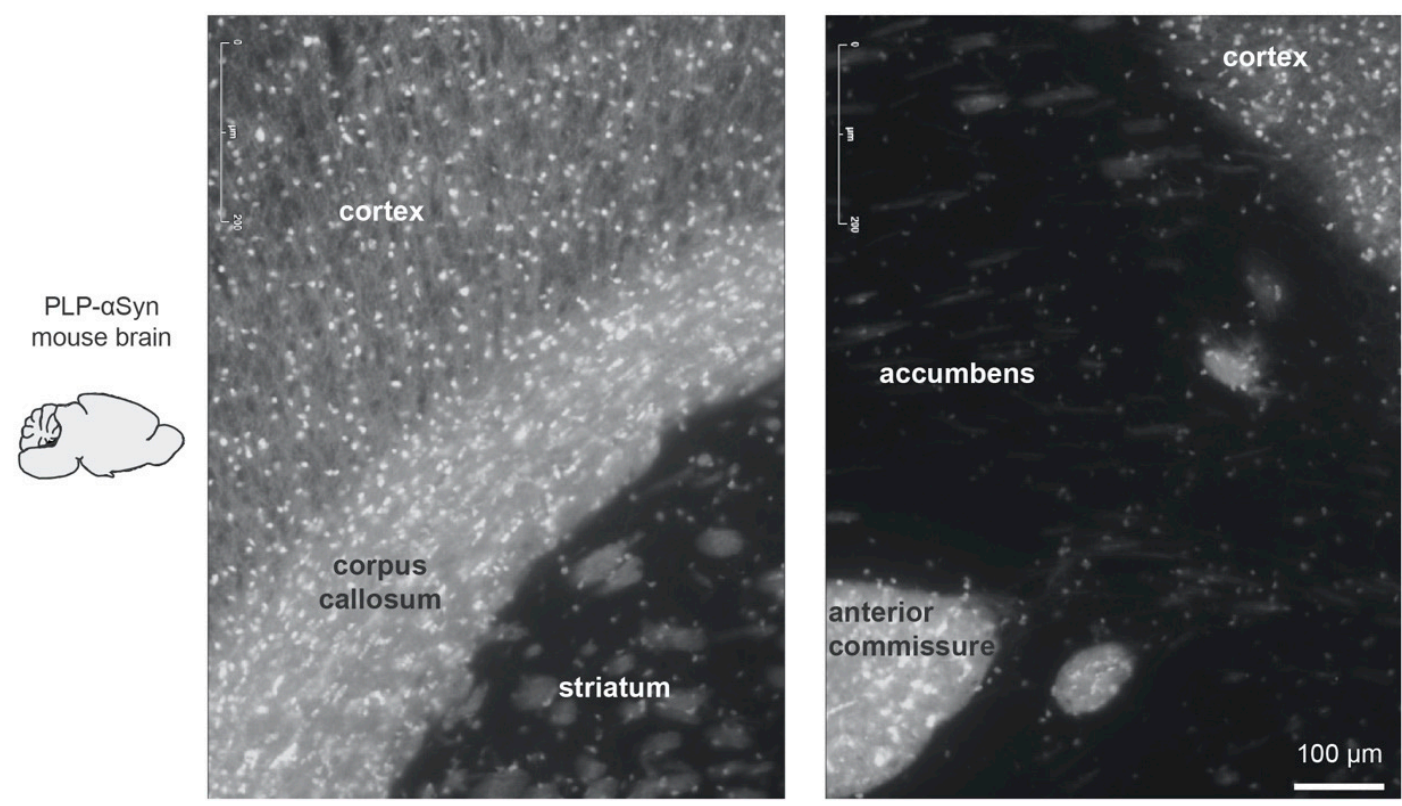

Figure 3. pS129-positive $\alpha$-syn mono/oligomers co-sediment with MBP and are enriched in myelinated regions of the PLP- $\alpha$ Syn mouse brain. (A) Sedimentation analysis of the different species of $\alpha$-syn found in WT or PLP- $\alpha$ Syn mouse brains with or without myelin floatation. Pooled 9 to 24 months-old mouse brain homogenates $(n=3)$ were subjected to SarkoSpin normal procedure (with sucrose cushion, bottom) or to the same procedure without myelin floatation sucrose cushion (without sucrose, top). The content in endogenous murine $\alpha$-syn (D37A6), total $\alpha$-syn (syn1), human $\alpha$-syn (MJFR1), pS129- $\alpha$-syn (EP1536Y) and myelin basic protein (MBP) of SarkoSpin supernatant and pellet fractions were assessed by filter trap followed by immunolabelling with the respective antibodies. Pictures are representative of $n=2$ independent Sarkospin procedures quantified in Supplementary Figure S3. (B) Representative pS129- $\alpha$-syn immunofluorescence staining of six months-old PLP- $\alpha$ Syn mouse brain sections. Hyperphosphorylated forms of the protein are prominent in regions with long myelinated axons such as the corpus callosum and the anterior commissure. 


\subsection{Is the Presence of Neuronal Human $\alpha$-syn in PLP- $\alpha$ Syn Primary Cortical Cultures due to an} OL-to-Neuron Transfer of the Protein?

In spite of the absence of fibrillar amyloid forms of $\alpha$-syn in their brains, the PLP- $\alpha$ Syn mice yet exhibit a partial neuronal depletion in the substantia nigra and the striatum (reviewed in Reference [24]) as well as lesions in brainstem nuclei such as the pre-Bötzinger complex [24] and have been reported to develop defects in the execution of motor tasks as well as autonomic dysfunctions $[25,28,29,34,37]$.

One possibility we thus considered was that a fraction of the $\alpha$-syn produced by the OLs could be transferred to neurons and could disturb their normal function. To understand if such a crosstalk between OLs and neurons could take place in these mice, we studied the $\alpha$-syn pools in primary cortical cultures containing OLs and neurons both derived from PLP- $\alpha$ Syn mice and compared it with primary cortical cultures from WT animals enriched with OLs isolated from PLP- $\alpha$ Syn mice (Figure 4). If a transfer of $\alpha$-syn from the OLs to the neurons was present, the protein should be observable in both types of cultures. Figure 4A shows that the OLs express high levels of human $\alpha$-syn (strongly syn1 positive, D37A6 negative) in DIV 30 fully differentiated primary cortical cultures from PLP- $\alpha$ Syn mice and that neurons endogenously express mouse $\alpha$-syn (barely syn1-positive with these imaging settings, D37A6 positive). Concomitant imaging of neurons with NeuN and of human $\alpha$-syn with MJFR1 in these cultures (Figure 4B) (and thresholding out the low unspecific nuclear signal yielded by MJFR1) indicated, however that a significant number of neurons $(\sim 15 \%)$ also contain human $\alpha$-syn (Figure 4B upper panel row, Figure 4C left panel, Figure 4E) compatible with the hypothesis of a transfer of human $\alpha$-syn from OLs to the neurons. However, DIV 30 cortical cultures from WT mice enriched with OLs from PLP- $\alpha$ Syn mice did not show signs of such a transfer, in spite of a strong expression of human $\alpha$-syn by the OLs from the transgenic animals (Figure 4B, lower panel row, Figure $4 \mathrm{C}$ right panel, Figure 4E). This indicates that the presence of human $\alpha$-syn in the neurons of PLP- $\alpha$ Syn mice is probably not due to a transfer from the OLs but rather to an endogenous expression leak of the PLP promoter in the neurons. Note that it could be argued that the human $\alpha$-syn found in neurons in the cultures from PLP- $\alpha$ Syn mice derives from a transfer having taken place beforehand, that is, during embryonic life, before the culture phase. This however seems unlikely because it takes several weeks in culture before the primary OLs start expressing human $\alpha$-syn (Supplementary Figure S4A) suggesting that the PLP promoter of the transgene is not significantly activated in OLs during embryonic life. It thus becomes conceivable that the neuronal pathology and dysfunction seen in the PLP- $\alpha$ Syn mice could be attributable, at least in part, to a direct neuronal effect due to a mis-targeted expression of human $\alpha$-syn in neurons. Note that in our conditions, the number of live neurons (NeuN positive) in the DIV 30 primary cortical cultures was similar for WT and PLP- $\alpha$ Syn mice and was not modified in the WT cultures enriched with OLs from PLP- $\alpha$ Syn mice (Figure 4D). 
A
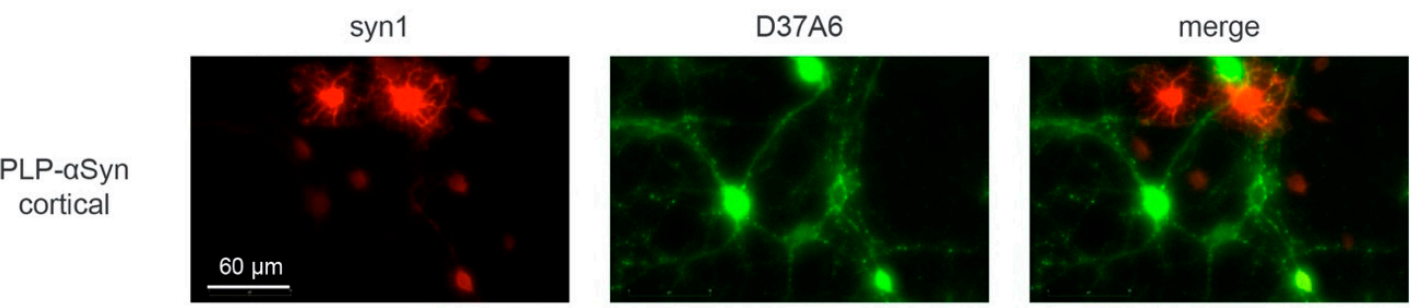

B

NeuN

MJFR1

merge
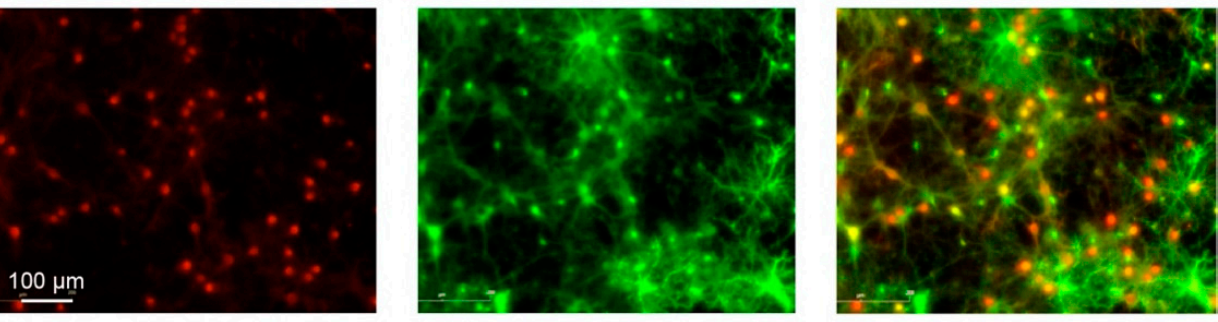

PLP-aSyn cortical
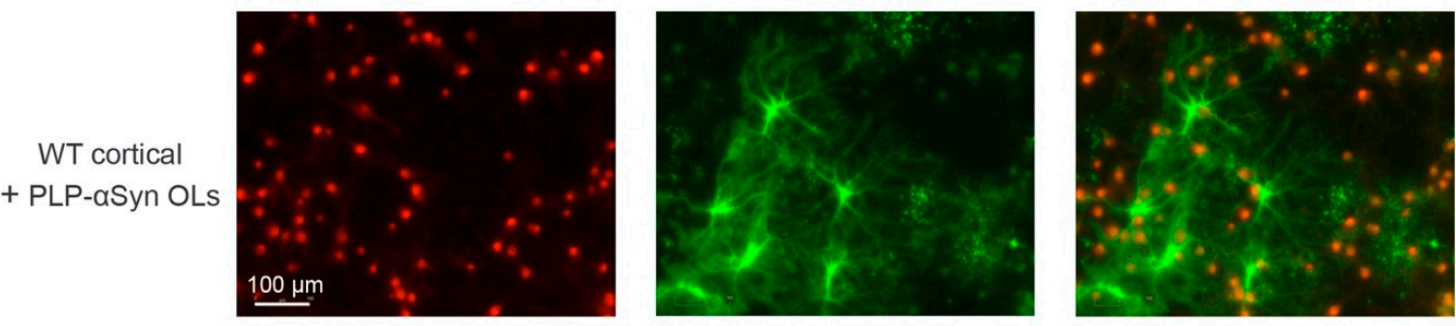

C
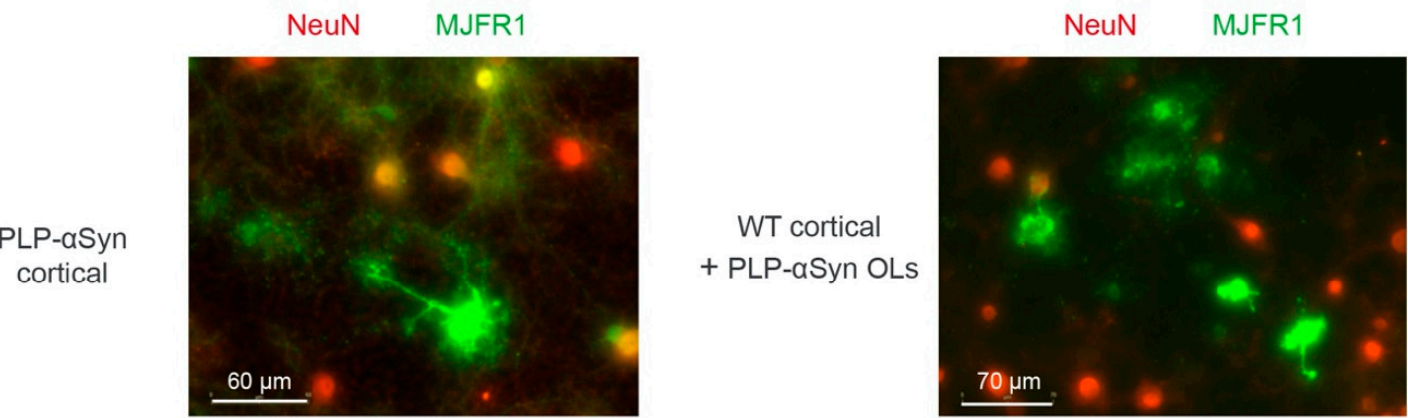

D

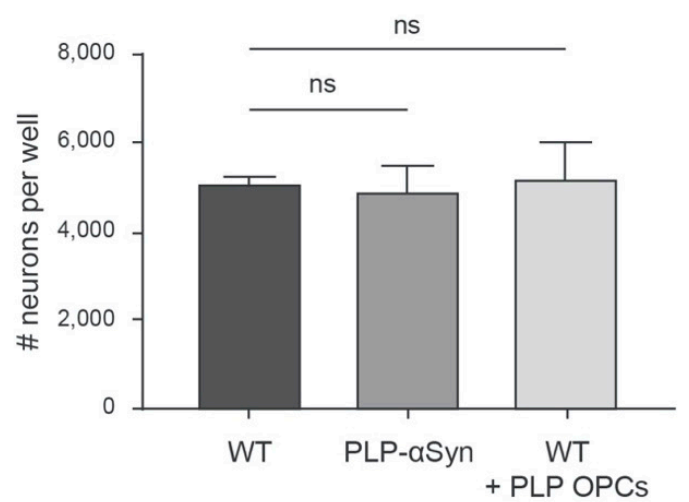

E

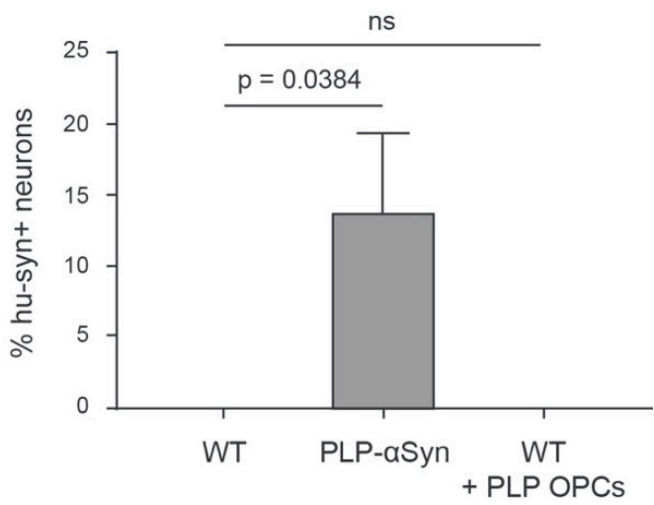

Figure 4. The presence of neuronal human $\alpha$-syn in PLP- $\alpha$ Syn primary cortical cultures is not due to an OL-to-neuron transfer of the protein. (A) Representative immunofluorescence imaging of total $\alpha$-syn (syn1, red) and endogenous murine $\alpha$-syn (D37A6, green) in primary cortical cultures PLP- $\alpha$ Syn mouse. (B) Representative immunofluorescence imaging of neurons (NeuN, red) and human $\alpha$-syn (MJFR1, green) in primary cortical cultures from PLP- $\alpha$ Syn mouse (top) or WT mouse supplemented with PLP- $\alpha$ Syn oligodendrocytes (bottom). (C) Close-up representative illustrations of the merged pictures shown in B. (D) Bar graph of the quantifications of the number of neurons (NeuN+ cells) per well. 
Equal averages of approximately 5000 neurons were obtained, with no significant differences between conditions (Holm-Sidak corrected multiple $t$-tests), ns: not statistically different. (E) Bar graph of the quantifications of the ratios of human $\alpha$-syn positive neurons (hu-syn $+\mathrm{NeuN}+/ \mathrm{NeuN}+$ cells) per well in the three different culture conditions. After thresholding out the residual unspecific nuclear staining yielded by MJFR1, these cells are detected solely in PLP- $\alpha$ Syn primary cortical cultures ( $p$-Values obtained with Holm-Sidak corrected multiple $t$-tests). For each condition 9 fields corresponding to $5.13 \mathrm{~mm}^{2}$ that is, $15 \%$ of the total well surface, of 2 independent wells were analyzed. Results are representative of 3 independent experiments.

\subsection{S129-Phosphorylated Human $\alpha$-Syn Is Enriched at "Hot Spots" in OL Processes}

In order to better understand how OLs from PLP- $\alpha$ Syn mice manage to accommodate exceptionally high levels of human $\alpha$-syn [34] without experiencing $\alpha$-syn fibrillization, we imaged the subcellular distribution of pS129-positive (i.e., monomers and oligomers) as well as the unphosphorylated $\alpha$-syn pools in purified OL cultures and cortical cultures from transgenic animals (Figure 5 and Supplementary Figure S4B). At DIV20, purified OLs express the differentiation marker CNPase (Figure 5A CNPase) with human $\alpha$-syn showing a corresponding diffuse cytoplasmic staining pattern (Figure 5A, MJFR1, merge) instead, and, reminiscent of what is also observed in melanoma cells [38], pS129-positive $\alpha$-syn exhibits a specific compartmentalized distribution pattern with "hot spots" delineating the tip of certain cellular processes as well as lamellipod-like plasma membrane regions (Figure 5B-C, EP1536Y, merge) [39]. This is particularly evident in OLs in which unphosphorylated and phosphorylated $\alpha$-syn forms were concomitantly revealed (Figure 5C, left and middle panels), with the additional observation of a variable association of pS129-positive $\alpha$-syn with the nucleus (Figure 5C, right panel).

Collectively, these results show that second to overexpression, fibrillization of $\alpha$-syn does not take place in PLP- $\alpha$ Syn mice and that the phosphorylated fraction of $\alpha$-syn remains monomeric and oligomeric and gets associated with the nucleus and with specialized regions of the cellular membrane [39]. This indicates that differentiated OLs that turned off their endogenous $\alpha$-syn expression [6], can accommodate the experimental overexpression of human $\alpha$-syn, phosphorylate monomers and oligomers and direct the latter in specific sub-cellular compartments. It is possible that our observations regarding PLP- $\alpha$ Syn mice could also hold true for CNP- and MBP- $\alpha$ Syn mice $[15,16]$ and, more generally, for other targeted $\alpha$-syn overexpression strategies. The fact that the fibrillization process of $\alpha$-syn does not take place in the OLs of PLP- $\alpha$ Syn mice despite very high $\alpha$-syn expression levels is particularly intriguing and should help in the identification of the signaling pathways capable of inducing $\alpha$-syn amyloid aggregation. 
A

WT OLs

PLP-aSyn

OLs

WT OLs

B
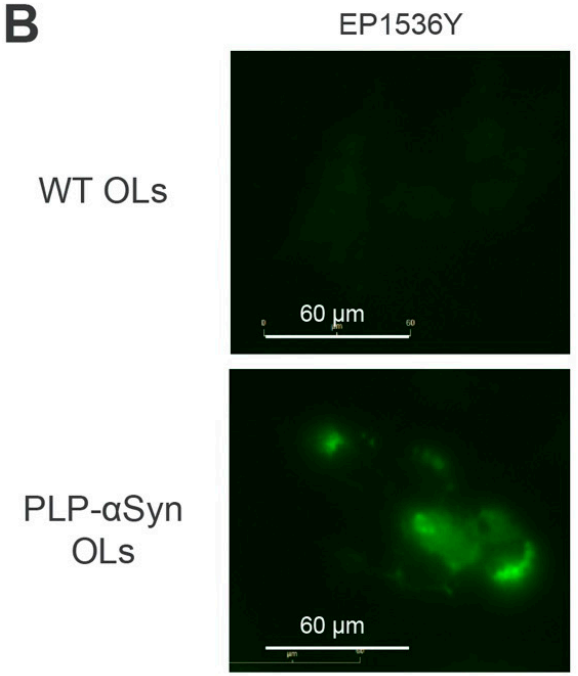

C
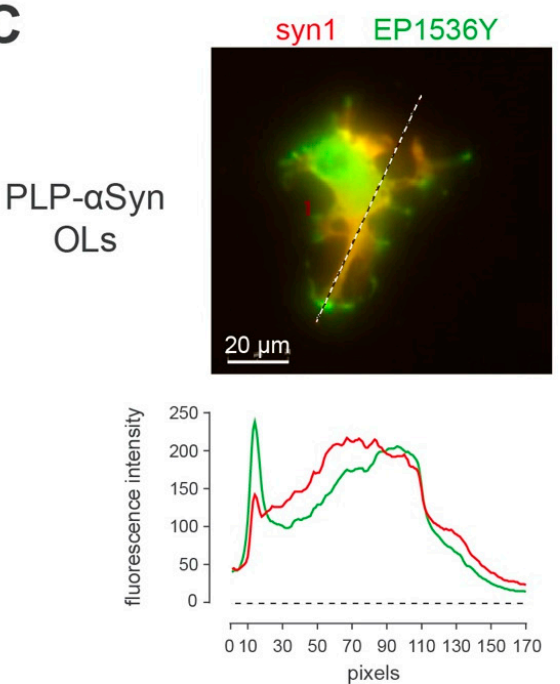

MJFR1
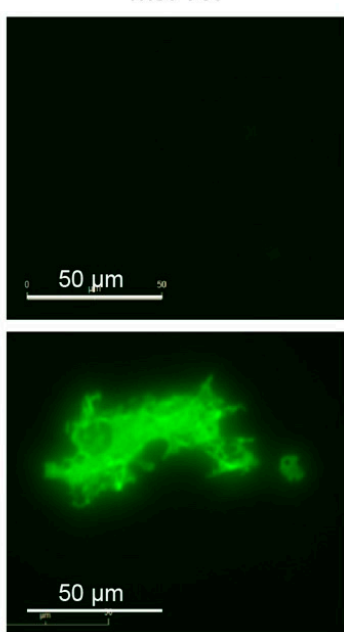
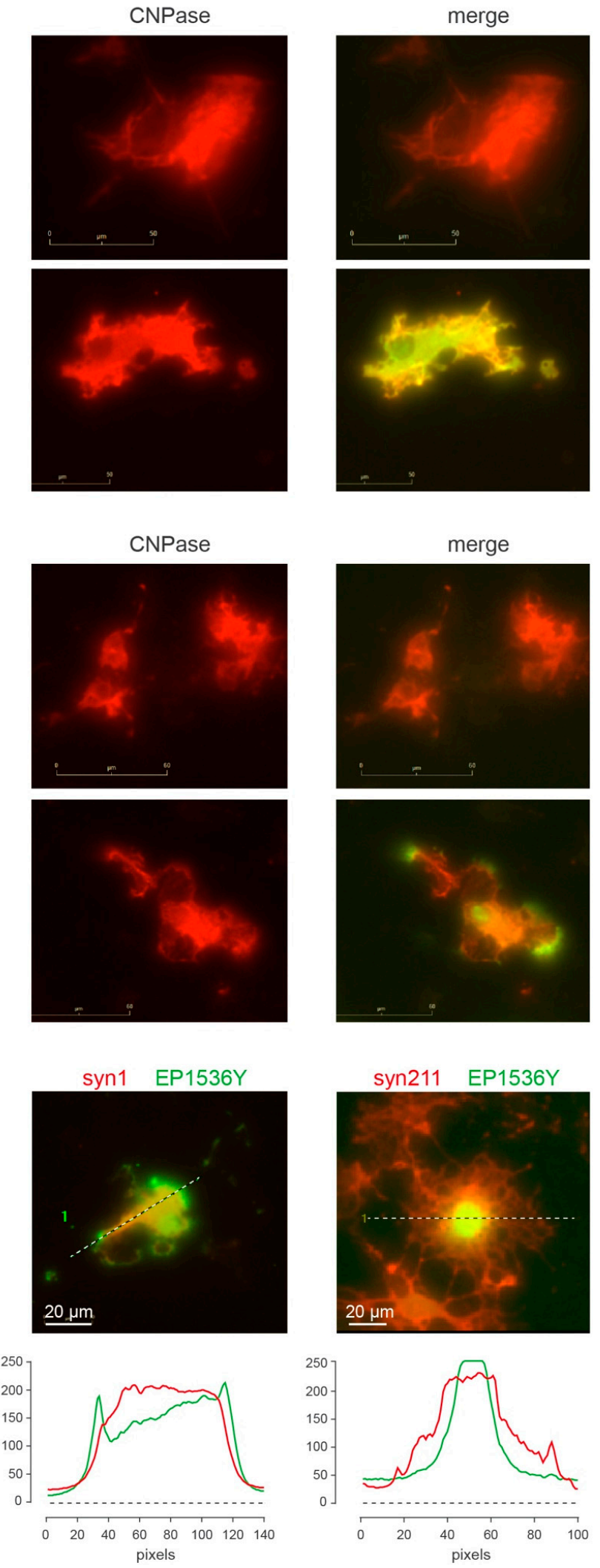

syn211 EP1536Y
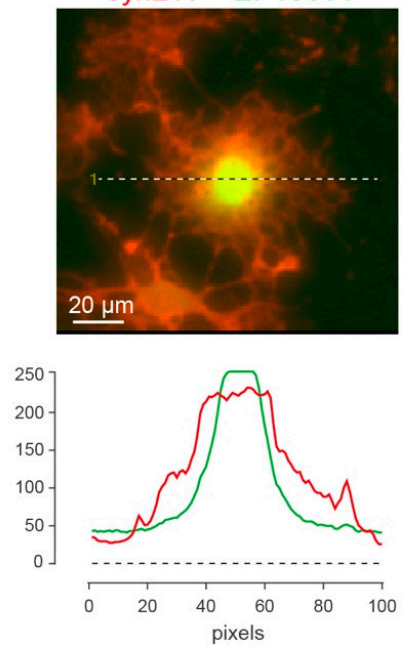

Figure 5. pS129 positive human $\alpha$-syn is enriched in OL processes (A) Representative immunofluorescence imaging of $2^{\prime}, 3^{\prime}$-cyclic-nucleotide $3^{\prime}$-phosphodiesterase (CNPase, red) together with human $\alpha$-syn (MJFR1, green) in primary cultures of OLs from PLP- $\alpha$ Syn mice and wt mice. (B) Representative immunofluorescence imaging of $2^{\prime}, 3^{\prime}$-cyclic-nucleotide $3^{\prime}$-phosphodiesterase 
(CNPase, red) together with pS129- $\alpha$-syn (EP1536Y, green, bottom) in primary cultures of OLs from PLP- $\alpha$ Syn mice and wt mice. (C) Left and middle panels: localization at the processes tips of phosphorylated human $\alpha$-syn, with total $\alpha$-syn (syn1, red) and pS129- $\alpha$-syn (EP1536Y, green) immunofluorescence staining and respective quantification of the profile intensities by linescan analysis. Right panel shows the occasional nuclear localization of phosphorylated human $\alpha$-syn, with total human $\alpha$-syn (Syn-211, red) and pS129- $\alpha$-syn (EP1536Y, green) immunofluorescence staining and respective quantification of the profile intensities by linescan analysis. Results are representative of 3 independent experiments.

3.5. Aggregation of Human $\alpha$-Syn Does Not Spontaneously Take Place on OLs from PLP- $\alpha$ Syn Mice but Can Be Experimentally Seeded with PFFs

Interestingly, we found that $\alpha$-syn amyloid aggregation could be induced in OLs by seeding the cortical cultures from PLP- $\alpha$ Syn mice with exogenous preformed fibrils made of human $\alpha$-syn (PFFs) (Figure 6) [30]. In Figure 6A, B we challenged the cultures with 2 different PFF production batches [30] (see also methods section) and observed a drastic morphological change of the MJFR1 signal distribution in OLs corresponding to a "contraction" compatible with an aggregation of $\alpha$-syn induced by the exogenous PFFs in these cells. As expected, PFF treatment induced a canonical pS129-positive neuronal synucleinopathy at the expense of endogenous $\alpha$-syn in WT cultures (Figure 6C, upper row panel and Supplementary Figure S5). In cultures enriched with OLs from PLP- $\alpha$ Syn mice (Figure 6C, lower row panel), PFFs induced a massive increase of the EP1536Y signal that coincided with a strong positivity with the aggregate-specific antibody synF1 [40]. This indicated that PFFs were able to seed the fibrillization process in OLs overexpressing $\alpha$-syn. Note that the neuronal aggregates seen in the upper panel row of Figure $6 \mathrm{C}$ are also synF1 positive but that the signal amplification chosen to document the process in OLs left this signal close to the background in the images shown. Using the topology of the EP1536Y signal, we performed a differential quantification of the fibrillization process induced by PFFs in neurons (Figure 6D) and in the OLs (Figure 6E) that confirmed this observation. For quantifying pS129-positive $\alpha$-syn in neurons, the dedicated Incucyte image analysis module allowing the specific segmentation of neuritic fluorescence signals was used. For quantifying aggregated $\alpha$-syn in OLs a double segmentation of cell bodies based on synF1 basal signal linked to overexpression and on EP1536Y was used.

The capability of PFFs to induce fibrillization in the OLs indicates that overexpression of $\alpha$-syn alone is not sufficient to trigger $\alpha$-syn nucleation but that the $\alpha$-syn pool derived from overexpression is available for templating by exogenous seeds.

In conclusion, while PLP- $\alpha$ Syn mice do not spontaneously develop a fibrillar synucleinopathy similar to MSA patients, their OLs can however be induced to do so by using exogenous PFFs as seeds. Interestingly, early imaging of the primary PLP- $\alpha$ Syn cultures only 4 days after seeding with the PFFs revealed that they decorated the surface of the neurons but not that of the OLs (Supplementary Figure S6) revealing possible different PFF uptake modes in the two cell types.

In agreement with a recent report concerning wild-type mice [41], intracerebral injections of PFFs in PLP- $\alpha$ Syn mice could thus represent a way to model a bona fide fibrillar synucleinopathy in OLs in vivo. Besides the development of such a model, next steps will also consist in understanding why $\alpha$-syn overexpression in the OLs of the PLP- $\alpha$ Syn mice results in the accumulation of phosphorylated monomers and oligomers instead of yielding a pathological burden of phosphorylated amyloid fibrils. 
A
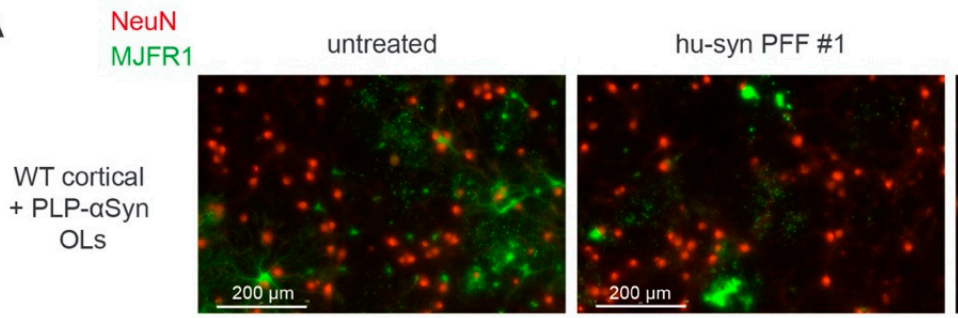

hu-syn PFF \#2

B

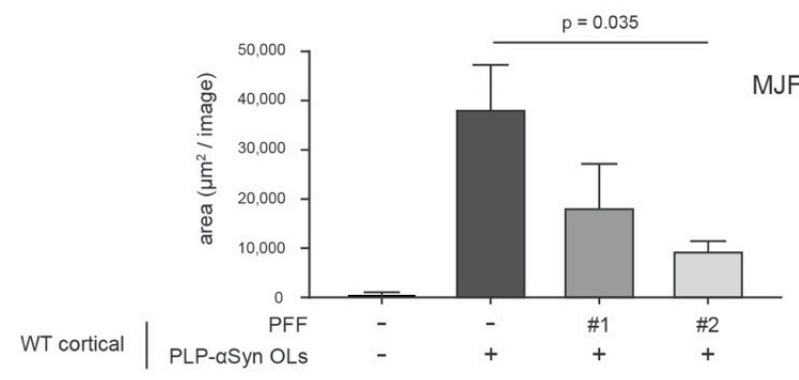

C $\quad{ }_{\text {EP1536Y }}^{\text {synf1 }}$

untreated

hu-syn PFF \#1

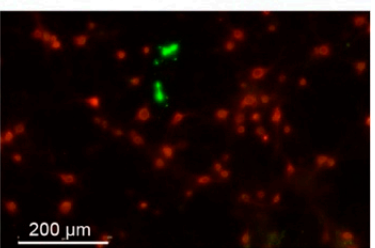

MJFR1 area
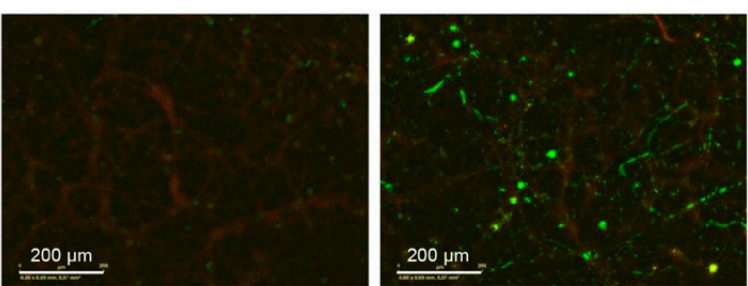

hu-syn PFF \#2

WT cortical

WT cortical + PLP-aSyn OLs
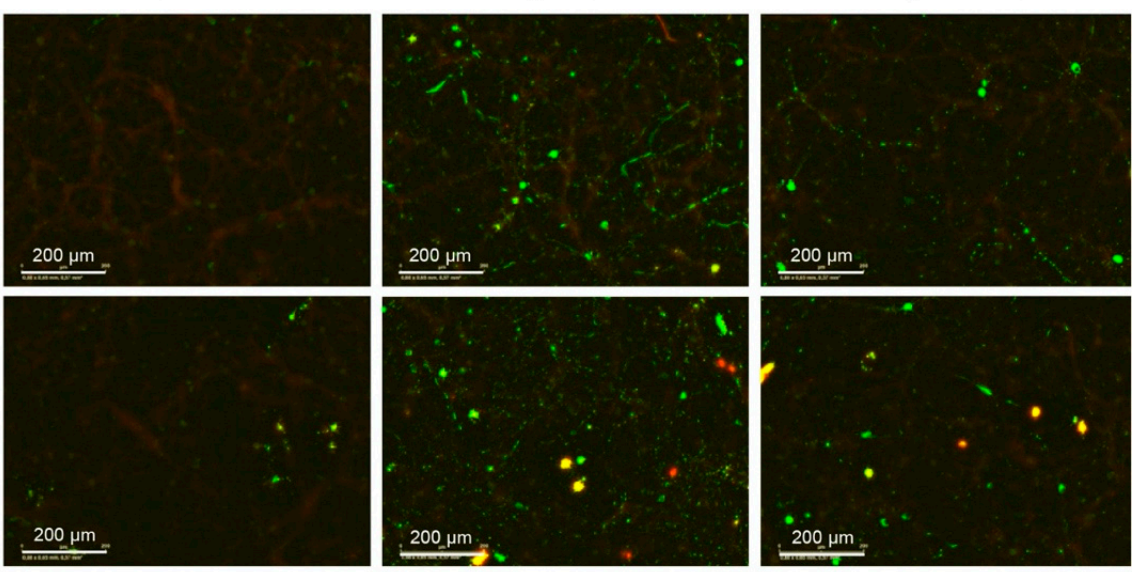

D
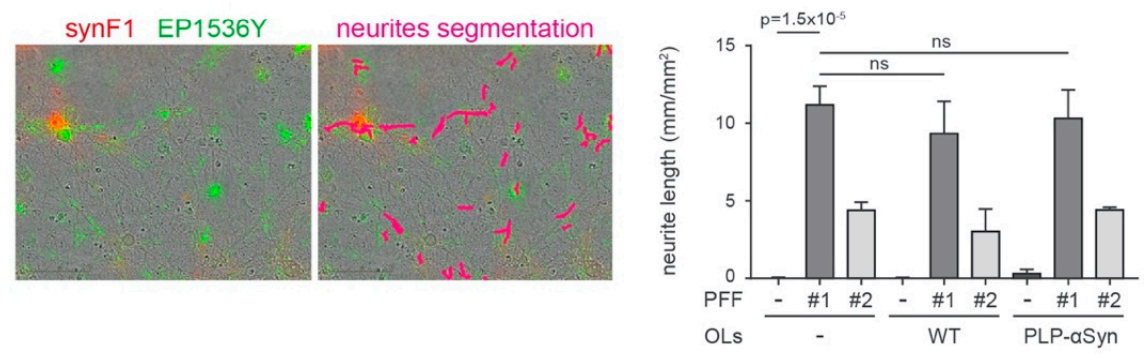

E

synF1 EP1536Y OLs segmentation
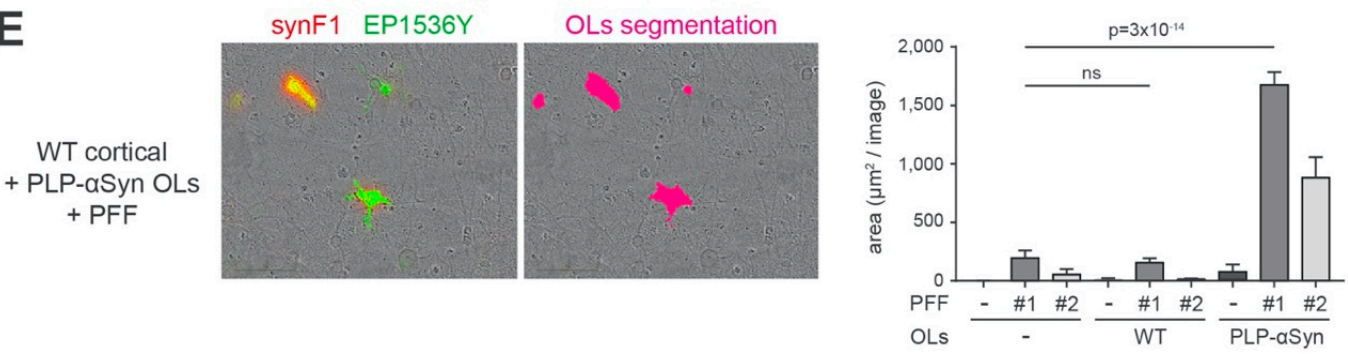

Figure 6. Aggregation of human $\alpha$-syn does not spontaneously take place on OLs from PLP- $\alpha$ Syn mice but can be experimentally seeded by challenging with PFFs. (A) Representative immunofluorescence imaging of neurons (NeuN, red) and human $\alpha$-syn (MJFR1, green) in primary cortical cultures from WT mouse supplemented with OLs from PLP- $\alpha$ Syn mice, untreated (left) or challenged with human $\alpha$-syn PFF of type 1 (middle, PFF \#1) or type 2 (right, PFF \#2). Pictures were taken four weeks post 
treatment and show OLs morphology changes with cellular shrinking upon PFF treatments. (B) Bar graph representing the quantification of MJFR1 area in the different conditions described in A, traducing the OLs surface measurements, with a significant shrinking of these cells upon challenge with PFF (pValues obtained with Holm-Sidak corrected multiple $t$-tests). (C) Representative immunofluorescence imaging of the synucleinopathy as shown by aggregated $\alpha$-syn (synF1, red) and hyperphosphorylated pS129- $\alpha$-syn (EP1536Y, green) in primary cortical cultures from WT mouse (top) and the same cultures supplemented with OLs from PLP- $\alpha$ Syn mice (bottom). These two cultures were untreated (left) or challenged with human $\alpha$-syn PFF of type 1 (middle, PFF\#1) or type 2 (right, PFF \#2). (D) Representative image shown in $\mathrm{C}$ with a neurite segmentation filter applied (right, pink), allowing the quantification of neuritic synucleinopathy plotted in the bar graph. Total synucleinopathy neurite length is shown for WT primary cortical cultures supplemented or not with WT or PLP- $\alpha$ Syn OLs and challenged or not with PFF of the two types. The extent of the neuronal synucleinopathy is independent of the addition of OLs ( $p$ values obtained with Holm-Sidak corrected multiple $t$-tests), ns: not statistically different. (E) Representative image shown in C with an OLs segmentation filter applied (right, pink), allowing the quantification of synucleinopathy located in the OL cell bodies, plotted in the bar graph. Total OLs synucleinopathy is shown as area measured for WT primary cortical cultures supplemented or not with WT or PLP- $\alpha$ Syn OLs and challenged or not with PFF of the two types ( $p$ values obtained with Holm-Sidak corrected multiple $t$-tests). For each condition 9 fields of 2 independent wells were analyzed corresponding to $5.13 \mathrm{~mm}^{2}$ that is, $15 \%$ of the total well surface. Results are representative of 2 independent experiments.

\subsection{Considerations about the Face vs. Predictive Value of the PLP- $\alpha$ Syn Mouse Model}

The new data presented here show that the $\alpha$-syn OL load present in the brains of PLP- $\alpha$ Syn mice cannot be compared to the GCIs that characterize MSA. The former is constituted of large amounts of phosphorylated monomers and oligomers that are neither observed in MSA or PD patients nor in control subjects (Figure 2 and Supplementary Figure S2), while the latter are filled with typical $\alpha$-syn amyloid fibrillar species that very well compare with the ones found in PD patients and with pure synthetic $\alpha$-syn fibrils (Figure 2 and Supplementary Figure S2).

It should be highlighted that our observations do not support the idea that GCIs or Lewy Bodies do not contain $\alpha$-syn amyloids [42]: instead, our analysis reveals the prominent presence of amyloid fibrils, which is in agreement with numerous previous studies $[35,36]$ but importantly, it also points to the absence of phosphorylated monomers and oligomers in the extracts of MSA and PD patients that could differentiate them from control subjects (Figure 2 and Supplementary Figure S2).

The absence of fibrillar $\alpha$-syn amyloid species in the brain of the PLP- $\alpha$ Syn mice seems to be in line with the notion put forward by certain authors that the clinical manifestations seen in these animals are "mild" [24,25] compared to MSA. In particular, while MSA is rapidly evolutive and causes the premature death of the patients, the lifespan of PLP- $\alpha$ Syn mice is normal [24]. This later observation is striking if one considers that their total brain $\alpha$-syn levels are 500 to $800 \%$ higher than in wild-type animals [34]. It thus seems a reasonable hypothesis to consider that the lack of formation of amyloid species in these transgenic mice can be responsible for the mildness of the clinical signs they exhibit.

According to our results and irrespective of their intensity, the clinical signs that develop in PLP- $\alpha$ Syn mice could thus have two non-mutually exclusive origins: (i) the existence of an expression leak in neurons that could cause direct detrimental effects in these cells and (ii) the steady presence of a high load of phosphorylated monomers and oligomers in the OLs leading to their progressive dysfunction. Whether this latter possibility could correspond to a mechanism mimicking a process taking place in MSA is not supported by our present data which indicate the absence of accumulation of phosphorylated monomers and oligomers in the extracts of MSA and PD patients compared to control subjects. It could be hypothesized that in the early stages of MSA, a transient peak of phosphorylated monomeric and oligomeric $\alpha$-syn species could precede and give way to the appearance of GCIs [13]. Whether clinical signs of MSA can be ascribed to the early and transient presence of such assemblies 
remains, however, to be explored. Thus, in spite of a documented face value of the PLP- $\alpha$ Syn mouse animal model, it appears premature to conclude on its predictive value.

Supplementary Materials: The following are available online at http://www.mdpi.com/2073-4409/9/11/2371/s1, Figure S1: Quantification of $\alpha$-syn species found in the different Sarkospin fractions, Figure S2: Human $\alpha$-syn and pS129- $\alpha$-syn sedimentation profiles in mouse or human brain and recombinant PFF samples, Figure S3: Quantification of different $\alpha$-syn species and MBP found in Sarkospin fractions, Figure S4: Human $\alpha$-syn is highly expressed in primary cultures of OLs from PLP- $\alpha$ Syn mice, Figure S5: Neuronal induced synucleinopathy upon challenging primary cortical cultures with human $\alpha$-syn PFF, Figure S6: Specific neuronal association of fibrillar $\alpha$-syn upon challenging primary cortical cultures with human $\alpha$-syn PFFs, Table S1: List of chemicals used the study.

Author Contributions: Conceptualization, F.D.G. and F.I.; methodology, F.L., X.H., F.D.G. and F.I.; validation, F.L., X.H., F.D.G. and F.I.; formal analysis, F.L., X.H., E.F., F.D.G. and F.I.; investigation, F.L., X.H., F.Z., E.D., E.F., F.D.G. and F.I.; biological resources, W.G.M. and E.B.; writing-original draft preparation, F.L., X.H., F.D.G. and F.I.; writing-review and editing, F.L., X.H., W.G.M., E.B., F.D.G. and F.I.; visualization, F.L., F.D.G. and F.I.; supervision, E.B., F.D.G. and F.I.; funding acquisition, E.B. All authors have read and agreed to the published version of the manuscript.

Funding: The project was conducted using financial support from the Region Nouvelle Aquitaine, the "Grand Prix" from the Del Duca foundation and the Innovative Medicines Initiative 2 Joint Undertaking under grant agreement No 116060 (IMPRiND). This Joint Undertaking receives support from the European Union's Horizon 2020 research and innovation program and EFPIA. This work is supported by the Swiss State Secretariat for Education, Research and Innovation (SERI) under contract number 17.00038. The opinions expressed and arguments employed herein do not necessarily reflect the official views of these funding bodies.

Acknowledgments: We thank Dr. P.O. Fernagut and Dr. B. Dehay for providing us with the PLP- $\alpha$ Syn mice and archival brain tissue, M. Deshors, Dr. P. Costet and Dr. C. Martin for animal care and breeding and Dr. M.L. Arotçarena for providing test histological material. We are grateful to the NeuroCEB that is run by a consortium of Patients Associations: ARSEP (association for research on multiple sclerosis), CSC (cerebellar ataxias) and France Parkinson for providing human brain samples.

Conflicts of Interest: The authors declare no conflict of interest.

\section{References}

1. Papp, M.I.; Kahn, J.E.; Lantos, P.L. Glial cytoplasmic inclusions in the CNS of patients with multiple system atrophy (striatonigral degeneration, olivopontocerebellar atrophy and Shy-Drager syndrome). J. Neurol. Sci. 1989, 94, 79-100. [CrossRef]

2. Papp, M.I.; Lantos, P.L. The distribution of oligodendroglial inclusions in multiple system atrophy and its relevance to clinical symptomatology. Brain 1994, 117, 235-243. [CrossRef] [PubMed]

3. Spillantini, M.G.; Crowther, R.A.; Jakes, R.; Cairns, N.J.; Lantos, P.L.; Goedert, M. Filamentous alpha-synuclein inclusions link multiple system atrophy with Parkinson's disease and dementia with Lewy bodies. Neurosci. Lett. 1998, 251, 205-208. [CrossRef]

4. Wakabayashi, K.; Yoshimoto, M.; Tsuji, S.; Takahashi, H. A-Synuclein Immunoreactivity in Glial Cytoplasmic Inclusions in Multiple System Atrophy. Neurosci. Lett. 1998, 249, 180-182. [CrossRef]

5. Meissner, W.G.; Fernagut, P.O.; Dehay, B.; Péran, P.; Le Traon, A.P.; Foubert-Samier, A.; Lopez Cuina, M.; Bezard, E.; Tison, F.; Rascol, O. Multiple System Atrophy: Recent Developments and Future Perspectives. Mov. Disord. 2019, 34, 1629-1642. [CrossRef]

6. Djelloul, M.; Holmqvist, S.; Boza-Serrano, A.; Azevedo, C.; Yeung, M.S.; Goldwurm, S.; Frisén, J.; Deierborg, T.; Roybon, L. Alpha-Synuclein Expression in the Oligodendrocyte Lineage: An in Vitro and in Vivo Study Using Rodent and Human Models. Stem Cell Rep. 2015, 5, 174-184. [CrossRef]

7. Asi, Y.T.; Simpson, J.E.; Heath, P.R.; Wharton, S.B.; Lees, A.J.; Revesz, T.; Houlden, H.; Holton, J.L. Alpha-synuclein mRNA expression in oligodendrocytes in MSA. Glia 2014, 62, 964-970. [CrossRef]

8. Song, Y.J.C.; Lundvig, D.M.S.; Huang, Y.; Wei, P.G.; Blumbergs, P.C.; Højrup, P.; Otzen, D.; Halliday, G.M.; Jensen, P.H. P25A Relocalizes in Oligodendroglia From Myelin To Cytoplasmic Inclusions in Multiple System Atrophy. Am. J. Pathol. 2007, 171, 1291-1303. [CrossRef]

9. Mavroeidi, P.; Arvanitaki, F.; Karakitsou, A.-K.; Vetsi, M.; Kloukina, I.; Zweckstetter, M.; Giller, K.; Becker, S.; Sorrentino, Z.A.; Giasson, B.I.; et al. Endogenous oligodendroglial alpha-synuclein and TPPP/p25 $\alpha$ orchestrate alpha-synuclein pathology in experimental multiple system atrophy models. Acta Neuropathol. 2019, 138, 415-441. [CrossRef] 
10. Yu, Z.; Shi, M.; Stewart, T.; Fernagut, P.-O.; Huang, Y.; Tian, C.; Dehay, B.; Atik, A.; Yang, D.; De Giorgi, F.; et al. Reduced oligodendrocyte exosome secretion in multiple system atrophy involves SNARE dysfunction. Brain 2020, 143, 1-18. [CrossRef]

11. Hasegawa, T.; Kikuchi, A.; Takeda, A. Pathogenesis of multiple system atrophy. Neurol. Clin. Neurosci. 2013, 1, 189-194. [CrossRef]

12. Reyes, J.F.; Rey, N.L.; Bousset, L.; Melki, R.; Brundin, P.; Angot, E. Alpha-synuclein transfers from neurons to oligodendrocytes. Glia 2014, 62, 387-398. [CrossRef] [PubMed]

13. Sekiya, H.; Kowa, H.; Koga, H.; Takata, M.; Satake, W.; Futamura, N.; Funakawa, I.; Jinnai, K.; Takahashi, M.; Kondo, T.; et al. Wide distribution of alpha-synuclein oligomers in multiple system atrophy brain detected by proximity ligation. Acta Neuropathol. 2019, 137, 455-466. [CrossRef] [PubMed]

14. Kahle, P.J.; Neumann, M.; Ozmen, L.; Müller, V.; Jacobsen, H.; Spooren, W.; Fuss, B.; Mallon, B.; Macklin, W.B.; Fujiwara, H.; et al. Hyperphosphorylation and insolubility of $\alpha$-synuclein in transgenic mouse oligodendrocytes. EMBO Rep. 2002, 3, 583-588. [CrossRef] [PubMed]

15. Shults, C.W.; Rockenstein, E.; Crews, L.; Adame, A.; Mante, M.; Larrea, G.; Hashimoto, M.; Song, D.; Iwatsubo, T.; Tsuboi, K.; et al. Neurological and neurodegenerative alterations in a transgenic mouse model expressing human $\alpha$-synuclein under oligodendrocyte promoter: Implications for multiple system atrophy. J. Neurosci. 2005, 25, 10689-10699. [CrossRef] [PubMed]

16. Yazawa, I.; Giasson, B.I.; Sasaki, R.; Zhang, B.; Joyce, S.; Uryu, K.; Trojanowski, J.Q.; Lee, V.M.Y. Mouse model of multiple system atrophy $\alpha$-synuclein expression in oligodendrocytes causes glial and neuronal degeneration. Neuron 2005, 45, 847-859. [CrossRef] [PubMed]

17. Heras-Garvin, A.; Weckbecker, D.; Ryazanov, S.; Leonov, A.; Griesinger, C.; Giese, A.; Wenning, G.K.; Stefanova, N. Anle138b modulates $\alpha$-synuclein oligomerization and prevents motor decline and neurodegeneration in a mouse model of multiple system atrophy. Mov. Disord. 2019, 34, 255-263. [CrossRef]

18. Bassil, F.; Fernagut, P.-O.; Bezard, E.; Pruvost, A.; Leste-Lasserre, T.; Hoang, Q.Q.; Ringe, D.; Petsko, G.A.; Meissner, W.G. Reducing C-terminal truncation mitigates synucleinopathy and neurodegeneration in a transgenic model of multiple system atrophy. Proc. Natl. Acad. Sci. USA 2016, 113, 9593-9598. [CrossRef]

19. Venezia, S.; Refolo, V.; Polissidis, A.; Stefanis, L.; Wenning, G.K.; Stefanova, N. Toll-like receptor 4 stimulation with monophosphoryl lipid A ameliorates motor deficits and nigral neurodegeneration triggered by extraneuronal $\alpha$-synucleinopathy. Mol. Neurodegener. 2017, 12, 1-13. [CrossRef]

20. Bassil, F.; Canron, M.H.; Vital, A.; Bezard, E.; Li, Y.; Greig, N.H.; Gulyani, S.; Kapogiannis, D.; Fernagut, P.O.; Meissner, W.G. Insulin resistance and exendin-4 treatment for multiple system atrophy. Brain 2017, 140, 1420-1436. [CrossRef]

21. Lopez-Cuina, M.; Guerin, P.A.; Canron, M.H.; Delamarre, A.; Dehay, B.; Bezard, E.; Meissner, W.G.; Fernagut, P.O. Nilotinib Fails to Prevent Synucleinopathy and Cell Loss in a Mouse Model of Multiple System Atrophy. Mov. Disord. 2020, 35, 1-11. [CrossRef]

22. Fujiwara, H.; Hasegawa, M.; Dohmae, N.; Kawashima, A.; Masliah, E.; Goldberg, M.S.; Shen, J.; Takio, K.; Iwatsubo, T. alpha-Synuclein is phosphorylated in synucleinopathy lesions. Nat. Cell Biol. 2002, 4, 160-164. [CrossRef] [PubMed]

23. Hasegawa, M.; Fujiwara, H.; Nonaka, T.; Wakabayashi, K.; Takahashi, H.; Lee, V.M.Y.; Trojanowski, J.Q.; Mann, D.; Iwatsubo, T. Phosphorylated $\alpha$-synuclein is ubiquitinated in $\alpha$-synucleinopathy lesions. J. Biol. Chem. 2002, 277, 49071-49076. [CrossRef] [PubMed]

24. Bleasel, J.M.; Halliday, G.M.; Kim, W.S. Animal modeling an oligodendrogliopathy-multiple system atrophy. Acta Neuropathol. Commun. 2016, 4, 12. [CrossRef] [PubMed]

25. Fernagut, P.O.; Meissner, W.G.; Biran, M.; Fantin, M.; Bassil, F.; Franconi, J.M.; Tison, F. Age-related motor dysfunction and neuropathology in a transgenic mouse model of multiple system atrophy. Synapse 2014, 68, 98-106. [CrossRef]

26. Stefanova, N.; Reindl, M.; Neumann, M.; Haass, C.; Poewe, W.; Kahle, P.J.; Wenning, G.K. Oxidative stress in transgenic mice with oligodendroglial $\alpha$-synuclein overexpression replicates the characteristic neuropathology of multiple system atrophy. Am. J. Pathol. 2005, 166, 869-876. [CrossRef]

27. Stemberger, S.; Poewe, W.; Wenning, G.K.; Stefanova, N. Targeted overexpression of human $\alpha$-synuclein in oligodendroglia induces lesions linked to MSA -like progressive autonomic failure. Exp. Neurol. 2010, 224, 459-464. [CrossRef] 
28. Kuzdas, D.; Stemberger, S.; Gaburro, S.; Stefanova, N.; Singewald, N.; Wenning, G.K. Oligodendroglial alpha-synucleinopathy and MSA-like cardiovascular autonomic failure: Experimental evidence. Exp. Neurol. 2013, 247, 531-536. [CrossRef]

29. Boudes, M.; Uvin, P.; Pinto, S.; Voets, T.; Fowler, C.J.; Wenning, G.K.; De Ridder, D.; Stefanova, N. Bladder dysfunction in a transgenic mouse model of multiple system atrophy. Mov. Disord. 2013, 28, 347-355. [CrossRef]

30. De Giorgi, F.; Laferrière, F.; Zinghirino, F.; Faggiani, E.; Lends, A.; Bertoni, M.; Yu, X.; Grélard, A.; Morvan, E.; Habenstein, B.; et al. Novel self-replicating $\alpha$-synuclein polymorphs that escape ThT monitoring can spontaneously emerge and acutely spread in neurons. Sci. Adv. 2020, 6, eabc4364. [CrossRef]

31. Laferrière, F.; Maniecka, Z.; Pérez-Berlanga, M.; Hruska-Plochan, M.; Gilhespy, L.; Hock, E.-M.; Wagner, U.; Afroz, T.; Boersema, P.J.; Barmettler, G.; et al. TDP-43 extracted from frontotemporal lobar degeneration subject brains displays distinct aggregate assemblies and neurotoxic effects reflecting disease progression rates. Nat. Neurosci. 2018, 22, 65-77. [CrossRef] [PubMed]

32. Pérez-Berlanga, M.; Laferrière, F.; Polymenidou, M. SarkoSpin: A Technique for Biochemical Isolation and Characterization of Pathological TDP-43 Aggregates. Bio-Protocol 2019, 9, 1-12.

33. Laferrière, F.; Tixador, P.; Moudjou, M.; Chapuis, J.; Sibille, P.; Herzog, L.; Reine, F.; Jaumain, E.; Laude, H.; Rezaei, H.; et al. Quaternary structure of pathological prion protein as a determining factor of strain-specific prion replication dynamics. PLoS Pathog 2013, 9, e1003702. [CrossRef]

34. Refolo, V.; Bez, F.; Polissidis, A.; Kuzdas-Wood, D.; Sturm, E.; Kamaratou, M.; Poewe, W.; Stefanis, L.; Angela Cenci, M.; Romero-Ramos, M.; et al. Progressive striatonigral degeneration in a transgenic mouse model of multiple system atrophy: Translational implications for interventional therapies. Acta Neuropathol. Commun. 2018, 6, 2. [CrossRef]

35. Schweighauser, M.; Shi, Y.; Tarutani, A.; Kametani, F.; Murzin, A.G.; Ghetti, B.; Matsubara, T.; Tomita, T.; Ando, T.; Hasegawa, K.; et al. Structures of $\alpha$-synuclein filaments from multiple system atrophy. Nature 2020, 585, 464-469. [CrossRef] [PubMed]

36. Lashuel, H.A. Do Lewy bodies contain alpha-synuclein fibrils? and Does it matter? A brief history and critical analysis of recent reports. Neurobiol. Dis. 2020, 141, 104876.

37. Flabeau, O.; Meissner, W.G.; Ozier, A.; Berger, P.; Tison, F.; Fernagut, P.O. Breathing variability and brainstem serotonergic loss in a genetic model of multiple system atrophy. Mov. Disord. 2014, 29, 388-395. [CrossRef] [PubMed]

38. Lee, B.R.; Matsuo, Y.; Cashikar, A.G.; Kamitani, T. Role of ser129 phosphorylation of $\alpha$-synuclein in melanoma cells. J. Cell Sci. 2013, 126, 696-704. [CrossRef]

39. Volles, M.J.; Lansbury, P.T., Jr. Relationships between the sequence of alpha-synuclein and its membrane affinity, fibrillization propensity and yeast toxicity. J. Mol. Biol. 2007, 366, 1510-1522. [CrossRef] [PubMed]

40. Vaikath, N.N.; Hmila, I.; Gupta, V.; Erskine, D.; Ingelsson, M.; El-Agnaf, O.M.A. Antibodies against alpha-synuclein: Tools and therapies. J. Neurochem. 2019, 150, 612-625. [CrossRef] [PubMed]

41. Uemura, N.; Uemura, M.T.; Lo, A.; Bassil, F.; Zhang, B.; Luk, K.C.; Lee, V.M.-Y.; Takahashi, R.; Trojanowski, J.Q. Slow progressive accumulation of oligodendroglial alpha-synuclein ( $\alpha$-syn) pathology in synthetic $\alpha$-syn fibril-induced mouse models of synucleinopathy. J. Neuropathol. Exp. Neurol. 2019, 78, 877-890. [CrossRef] [PubMed]

42. Shahmoradian, S.H.; Lewis, A.J.; Genoud, C. Lewy pathology in Parkinson's disease consists of crowded organelles and lipid membranes. Nat. Neurosci. 2019, 22, 1099-1109. [CrossRef] [PubMed]

Publisher's Note: MDPI stays neutral with regard to jurisdictional claims in published maps and institutional affiliations.

(C) 2020 by the authors. Licensee MDPI, Basel, Switzerland. This article is an open access article distributed under the terms and conditions of the Creative Commons Attribution (CC BY) license (http://creativecommons.org/licenses/by/4.0/). 